\title{
Multiuser MISO Indoor Visible Light Communications
}

\author{
A Thesis \\ Presented to \\ the Faculty of the School of Engineering and Applied Science \\ UNIVERSITY OF VIRGINIA \\ In Partial Fulfillment \\ of the Requirements for the Degree \\ Master of Science in Electrical Engineering \\ by \\ JIE LIAN \\ August 2014
}




\section{APPROVAL SHEET}

The thesis

is submitted in partial fulfillment of the requirements

for the degree of

Master of Science

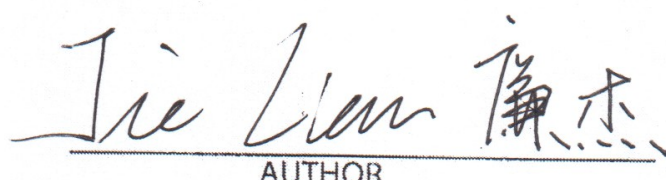

The thesis has been read and approved by the examining committee:

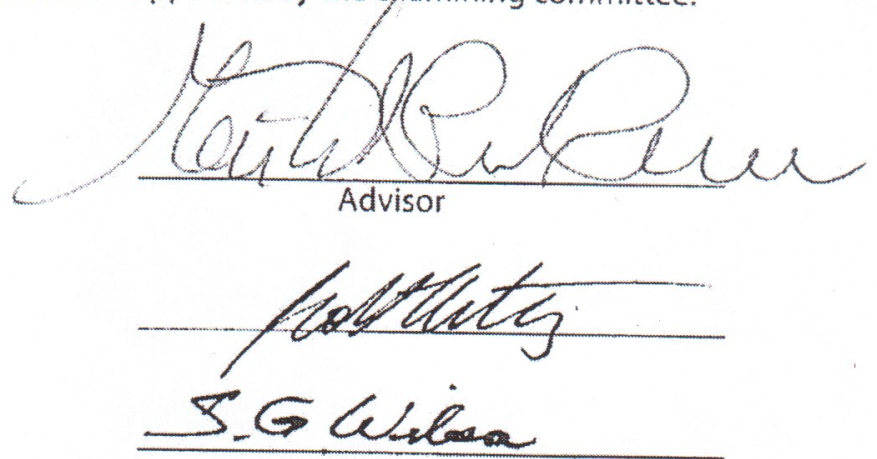

Accepted for the School of Engineering and Applied Science:

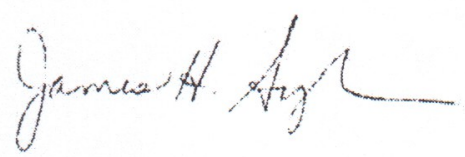

Dean, School of Engineering and Applied Science

August

2014 


\section{Acknowledgements}

I would like to gratefully and sincerely thank my advisor, Professor Maite BrandtPearce for her guidance, understanding, patience, and most importantly, persistent encouragement and continuous support during my graduate studies at the University of Virginia. Her deep knowledge of communication theory and her impeccable attitude towards research have greatly helped me to form a rigorous, dedicated, and creative research style for my future career life in the communication engineering field.

It is a blessing to be surrounded by many colleagues and friends at the University of Virginia. I am grateful to all my friends in Charlottesville and for making my life at U.Va. enjoyable.

I am grateful to my parents for their patience, help and love. "A journey of one thousand miles begins with one step," my parents always encourage me to take the first step. During this two years, my parents gave me the power to finish the master's program. 


\section{Abstract}

High speed data communication is an essential requirement for future indoor wireless networks. Visible light communication (VLC) is a strong competitor for highspeed wireless networking since it brings an energy efficient and cost effective solution for indoor connectivity by integrating the internet access points with the illumination system. In this thesis we analyze the characteristics of a channel model for indoor VLC systems and consider the one user case using an M-ary pulse amplitude modulation (M-PAM) scheme. On that basis, we then focus on the multiuser case, for which we propose a power allocation joint optimization (PAJO) algorithm that maximizes the minimum signal to interference pulse noise ratio (SINR) of all users. We design a minimum mean square error (MMSE) filter to be used at the receiver

of each user. Using simulation results, we show that our proposed PAJO has better BER performance than previous proposed methods while satisfying the illumination requirements for all users in this system. 


\section{Contents}

1 Introduction 1

1.1 Research Motivation . . . . . . . . . . . . . . . 2

1.2 Literature Review . . . . . . . . . . . . . . . . . . . 4

1.3 Thesis Outline . . . . . . . . . . . . . . . . 5

2 Transmitter Model and Indoor VLC Channel Model 6

2.1 Transmitter Model . . . . . . . . . . . . . . . . . . 6

2.2 Indoor VLC Channel Model . . . . . . . . . . . . . . . . . . 8

2.3 Numerical Results of the Indoor Channel . . . . . . . . . . . . . . . . 12

3 One User VLC System 21

3.1 Signal to Noise Ratio and Bit Error Rate Analysis for On-Off Keying 21

3.2 M-PAM Modulation Scheme . . . . . . . . . . . . . . 24

3.3 Simulation Results of One User Case in Indoor VLC Systems . . . . . 27

4 Multiuser VLC System 31

4.1 Optical CDMA . . . . . . . . . . . . . . . . . 31

4.2 VLC MISO Technology . . . . . . . . . . . . . . . . . 34

4.3 Power Allocation Joint Optimization . . . . . . . . . . . . . 36

4.3.1 Multiuser MISO System Description . . . . . . . . . . . 36 
4.3.2 Other Power Allocation Systems . . . . . . . . . . . . . . . . . 42

4.3.3 Illumination Requirements . . . . . . . . . . . . . . . . . . 42

4.3.4 Numerical Results of PAJO . . . . . . . . . . . . . . 43

4.4 Chapter Summary . . . . . . . . . . . . . . . . 52

5 Conclusions and Future Work $\quad 54$

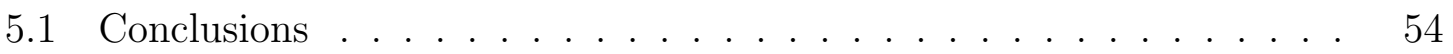

5.2 Future Work . . . . . . . . . . . . . . . . 55 


\section{List of Figures}

1.1 Normalized power/unit wavelength for optical wireless spectrum and ambient light sources $[1] \ldots \ldots \ldots$. . . . . . . . . . . . 2

2.1 1-LED lamp . . . . . . . . . . . . . . . . . . 7

2.2 Commonly used LED array $[26] \ldots \ldots$. . . . . . . . . . . 7

2.3 (a) 25-LED lamp structure from the side view, (b) 25-LED lamp structure from the bottom view . . . . . . . . . . . . . . 8

2.4 Basic indoor VLC channel model . . . . . . . . . . . . . . . . 8

2.5 Light rays classification . . . . . . . . . . . . . . . . . . . 9

2.6 LOS light rays model . . . . . . . . . . . . . . . . . . . . 11

2.7 Angle between radiation direction and user . . . . . . . . . . . 13

2.8 Indoor environment . . . . . . . . . . . . . . . . . . . . . 14

2.9 Illuminance (LOS and diffuse) distribution of 1-LED lamp with semiangle $=10^{\circ}$, $\operatorname{in} \operatorname{lux} \ldots \ldots \ldots \ldots \ldots \ldots \ldots$

2.10 Illuminance (LOS and diffuse) distribution of 1-LED lamp with semiangle $=20^{\circ}$, $\operatorname{in} \operatorname{lux} \ldots \ldots \ldots \ldots \ldots \ldots$

2.11 Illuminance (LOS and diffuse) distribution of 1-LED lamp with semiangle $=30^{\circ}$, $\operatorname{in} \operatorname{lux} \ldots \ldots \ldots \ldots \ldots \ldots$ 
2.12 Illuminance (LOS and diffuse) distribution of 25-LED lamp with semiangle $=3^{\circ}$, $\operatorname{in} \operatorname{lux} \ldots \ldots \ldots \ldots \ldots \ldots \ldots$

2.13 Illuminance (LOS and diffuse) distribution of 25-LED lamp with semiangle $=5^{\circ}$, $\operatorname{in} \operatorname{lux} \ldots \ldots \ldots \ldots \ldots \ldots \ldots$

2.14 Illuminance (LOS and diffuse) distribution of 25-LED lamp with semiangle $=10^{\circ}$, in $\operatorname{lux} \ldots \ldots \ldots \ldots \ldots \ldots$

2.15 Illuminance (LOS and diffuse) distribution of 25-LED lamp with semiangle $=20^{\circ}$, $\operatorname{in} \operatorname{lux} \ldots \ldots \ldots \ldots \ldots \ldots$

2.16 Illuminance (LOS and diffuse) distribution of 25-LED lamp with semiangle $=30^{\circ}$, $\operatorname{in} \operatorname{lux} \ldots \ldots \ldots \ldots \ldots$

2.17 Normalized impulse response of 1 -LED lamp with semiangle $=30^{\circ}$ at $(1.25,0.625,0) \ldots \ldots \ldots \ldots$

2.18 Normalized impulse response of 25 -LED lamp with semiangle $=30^{\circ}$ at $(1.25,0.625,0)$

3.14 -PAM modulation . . . . . . . . . . . . . . . 25

3.2 Block diagram of M-PAM schemes . . . . . . . . . . . . . 26

3.3 Optimal threshold with thermal noise . . . . . . . . . . . 26

3.4 BER distribution of 4-PAM modulation using the 25-LED lamp with semiangle $=5^{\circ} \ldots \ldots \ldots \ldots$. . . . . . . . . . . . . . . . . . 28

3.5 BER distribution of 4-PAM modulation using the 25-LED lamp with semiangle $=10^{\circ} \ldots \ldots \ldots \ldots \ldots$. . . . . . . . . . . . . . . . 28

3.6 BER distribution of 4-PAM modulation using the 25-LED lamp with semiangle $=20^{\circ} \ldots \ldots \ldots \ldots \ldots \ldots \ldots$

3.7 Theoretical BER performance and simulation BER performance of one user for 2-PAM, 4-PAM and 8-PAM for the 1-LED model . . . . . . 
4.1 OOC $\{1,2,4\}$ with length of $7 \ldots \ldots$. . . . . . . . . . . 33

4.2 Block diagram of a typical VLC MISO system . . . . . . . . . . . . . 34

4.3 Block diagram of the proposed PAJO algorithm to support $K$ users simultaneously. . . . . . . . . . . . . . . . . . . 36

4.4 BER of 1-LED lamp case with 1 to 4 users for 4 lamps, no illumination requirements, semiangle $=20^{\circ} \ldots \ldots \ldots$. . . . . . . . . . 45

4.5 BER of 25-LED lamp case with 1 to 4 users for 4 lamps, no illumination requirements, semiangle $=20^{\circ} \ldots \ldots \ldots$. . . . . . . . . 46

4.6 BD algorithm vs PAJO for 4, 3 and 2 users with 1-LED lamp case, no illumination requirements, semiangle $=20^{\circ} \ldots$. . . . . . . . . . . 46

4.7 BER of 4 users for 1-LED lamp case and 25-LED lamp cases with and without illumination requirements, the illumination is $400 \mathrm{~lx}$ with $10 \%$ tolerance . . . . . . . . . . . . . . . .

4.8 Illumination distribution comparison of (a) data transmission case and (b) no data transmission case. The red dots identify the real users, and the blue dots represent the virtual users with $10 \%$ tolerance, semiangle $=20^{\circ}$, and the 25-LED model. . . . . . . . . . . . . . . . . . 48

4.9 BER comparison of different tolerance with 4 users and 16 virtual users for the 25-LED lamp case, $400 \mathrm{~lx}$ illumination requirements . . . . . .

4.10 BER comparison of different semiangles and different tolerance with 4 users and 16 virtual users for 25-LED lamp case, 400 lx illumination requirements . . . . . . . . . . . . . . . . . 50 50

4.11 BER comparison of different number of virtual users for 25-LED lamp case, 400 lx illumination requirements and $10 \%$ tolerance . . . . . . 51

4.12 BER of OOC vs C-MEPPM without illumination constraints . . . . . 52 


\section{List of Tables}

3.1 Parameters and Estimates of Noise Variance . . . . . . . . . . . 24

4.1 OOC $(L, 3,1)$ sequence indexes for various length $\ldots . \ldots . \ldots . . . .33$

4.2 Parameters of Numerical Results of PAJO . . . . . . . . . . . . . . 44

4.3 Parameters of real design . . . . . . . . . . . . . . 53 


\section{Chapter 1}

\section{Introduction}

Visible light is defined as the light that is perceptible by the human eye, such as the light from the sun (red, orange, yellow, green, blue, violet, and so on), which has a wavelength ranging from $380 \mathrm{~nm}$ to $780 \mathrm{~nm}$. Figure 1.1 shows the visible light spectrum. From this figure, the visible light actually occupies a very small portion of the spectrum. Beyond this, there is also invisible light such as infrared and ultraviolet.

With the increasing demand for indoor communications, optical wireless communication started to become a popular research topic in both academia and industry [1]. Visible light communications, proposed by Nakagawa in 2000, uses the lighting system to provide short distance wireless communication links [2]. In this system, Nakagawa proposes LEDs as the light source and data transmitters. Comparing with RF and other optical wireless communication systems, the main advantages of VLC system using LEDs are:

- The LEDs have high illumination with low power consumption.

- LEDs have long life expectancy.

- VLC systems have higher privacy than RF communications. 


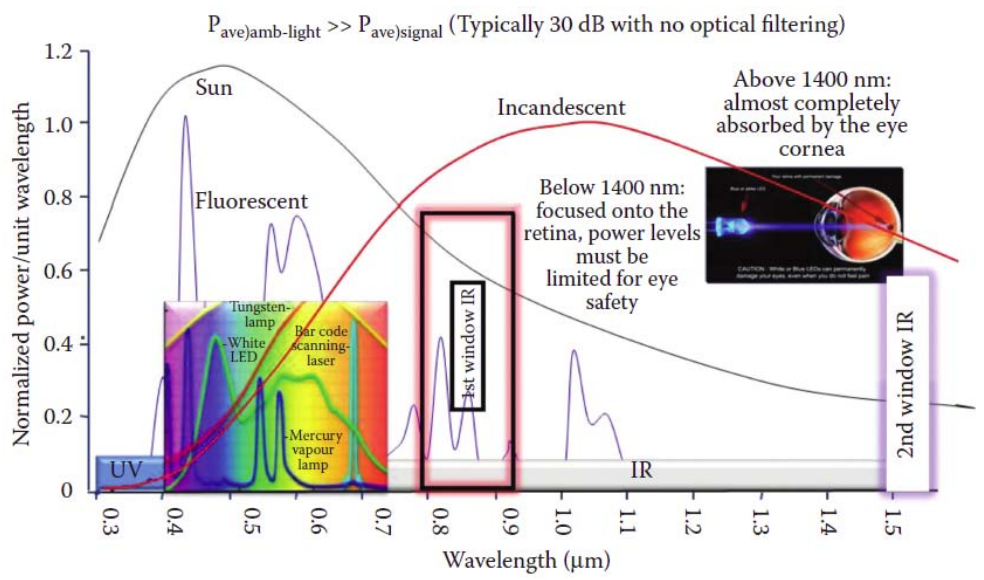

Figure 1.1: Normalized power/unit wavelength for optical wireless spectrum and ambient light sources [1]

- Visible light is safe for the human eye.

- LEDs have high on-off modulation frequency.

- LEDs are mercury free.

Because of these advantages, indoor optical wireless communications using LEDs is expected to be the trend in the future [1]. Nowadays, LEDs are used for many applications such as indoor illumination, automobile tail lights, advertising boards, traffic lights, and portable electronic devices such as cellphones and personal digital assistants (PDAs).

Visible light communication (VLC) is a novel communication system that uses white LEDs that are already being used for illumination to also transmit data. This technology is called Li-Fi, which stands for light fidelity [8] [9].

\subsection{Research Motivation}

Nowadays, VLC can be used in indoor one-user environments [5]. Because of the high privacy and low power consumption, more and more attention is paid to VLC. 
But sometimes there are more than one user in VLC systems; we cannot just consider the one-user case. Thus the multiuser case will be considered in this thesis. In this thesis, we employ multiple input single output (MISO) technology in our work. MISO is a specific case of multiple input multiple output (MIMO) technology. In MIMO system, there are more than one antennas (detectors) for each user. But for MISO system, there is only one antenna (detector) for each user. Therefore, we can get MISO from MIMO system.

Providing data transmission for multiple users is a very important concern. A significant aspect for a communication system is the quality of communication for multiple users simultaneously. Many scientists are doing research on providing data transmission for the multiuser case. [10] employs a MIMO technique and zero forcing algorithm to provide multiple access. A MIMO technique and block diagonalization algorithm are used to reduce the multiuser interference in [13]. [21] uses precoding and zero forcing algorithms to maximize the data rate of multiple users. But the researchers above did not consider the illumination requirements within the indoor space. Since the VLC system is a dual system for communication and lighting, satisfying the illumination requirements is of concern to us.

From this point of view, providing multiuser data transmission and illumination is our proposed topic. For communication purposes, the VLC system needs to provide users high quality wireless access. On the other hand, for illumination purpose, the LED lamps need to provide indoor illumination. To implement VLC systems, we need to achieve these two goals at the same time. 


\subsection{Literature Review}

In 2001, Yuichi Tanaka, Masao Nakagawa of Keio University and Shinichirou Haruyama at the SONY Computer Science Research Institute proposed the idea of visible light communications using white light LEDs to transmit signals [2-6]. They derived the relation between distance and performance of the receivers.

M-ary pulse amplitude modulation (M-PAM) is a modulation scheme suitable for optical communications because of its simple transmitter and receiver structure [15]. In VLC systems, a 16-level PAM scheme was employed to transmit video data at 40 $\mathrm{Mbit} / \mathrm{s}$ in [16].

After that, research in VLC has developed very fast and many techniques have been used in VLC, such as MIMO and orthogonal frequency-division multiplexing (OFDM) technique. Researchers have begun to consider how to implement MIMO technology in VLC [11] [29]. To increase the data rate, researchers combine MIMO technology and spatial modulation in [11]. Some researchers in Australia proposed an algorithm to implement an OFDM technique in VLC system to increase the transmission data rate $[12]$.

To provide data transmission for multiple users and to reduce the multiuser interference, researchers at the Georgia Institute of Technology proposed a precoding algorithm to support multiuser communications in VLC [21]. They focused on maximizing the sum data rates. However, in our work, we would like to reduce the multiuser interference and maximize the minimum signal to interference plus noise ratio (SINR) of all users to increase throughput and fairness among users. Some researchers from China proposed a precoding MIMO algorithm to support multiple users and reduce the multiuser interference [13]. We compare the performance of our

proposed algorithm with theirs in this thesis. Since [21] aims to improve the data 
rate and we focus on maximizing the SINR, we did not compare to [21] in this thesis.

In addition, optical code division multiple access (CDMA) technology has been considered for indoor VLC system to provide multiple access. Deriving a good code for indoor VLC systems is a critical challenge. Researchers in [22] proposed a novel optical CDMA coded multilevel expurgated pulse position modulation (MEPPM) code for VLC that could be used to adjust the illumination level in the indoor environment and also could be used for multiple access [24]. We adapt our precoding technique to use their coding method.

\subsection{Thesis Outline}

In this thesis, we analyze the transmitter model and channel model. After that, we consider the M-PAM modulation for a single user in a VLC system. Our research then addresses multiuser access in an indoor VLC environment. A new power allocation joint optimization (PAJO) algorithm is proposed. This algorithm provides optimal power allocation scheme for transmitters to maximize the minimum the SINR of all users using the minimum mean square error (MMSE) receiver for each user.

The rest of the thesis is organized as follows. In Chapter 2, we analyse the channel model characteristics and evaluate the performance using simulations. Chapter 3 considers a one user system for indoor VLC. We use M-PAM modulation scheme for one user in this system and analyze the BER performance of 2-PAM, 4-PAM and 8-PAM. Chapter 4 addresses the multiuser system in indoor VLC environments. In this chapter, we propose the PAJO algorithm, analyze the performance of PAJO and compare the performance of our proposed algorithm with what other researchers proposed in [13]. Chapter 5 summarizes the whole thesis and points out opportunities for some future work. 


\section{Chapter 2}

\section{Transmitter Model and Indoor VLC Channel Model}

Before discussing the performance of the VLC system, we need to analyze the indoor VLC channel model, (characteristics of the channel and the transmitter models). From the transmitter and channel models, we can obtain the channel gain for different users, the impulse response, and the power distributions on the floor. They will help us evaluate the performance of the indoor VLC system and design our system.

\subsection{Transmitter Model}

The power received at the users depends on both the transmitter model and the channel model. In this section, we describe two transmitter models. One is referred to as a 1-LED lamp model, and the other is a 25-LED lamp model.

The 1-LED lamp model is a transmitter model with only one LED in the lamp as shown in Figure 2.1. In this model, the radiation direction of the LED is straight down towards the floor. Although the most commonly used lamp for illumination purpose is an LED array as shown in Figure 2.2, and since the every LED in this 


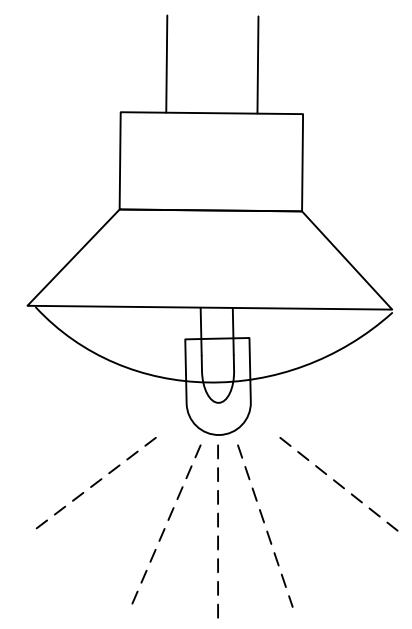

Figure 2.1: 1-LED lamp

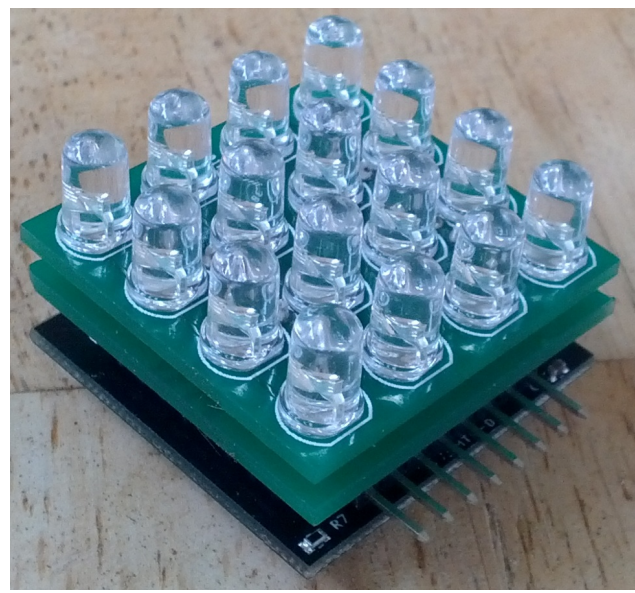

Figure 2.2: Commonly used LED array [26]

array is straight down towards the floor and the size of the LED array is relative small compared with the size of the indoor environment, the LED array can be modeled as a 1-LED lamp.

To cover more illumination area, and provide more power to the corner areas, we propose a 25-LED lamp model. The 25-LED lamp model is a transmitter consisting of 25 LEDs with different inclination angles. The LEDs in this lamp are deployed in three layers with 1, 8 and 16 LEDs as shown in Figure 2.3. 


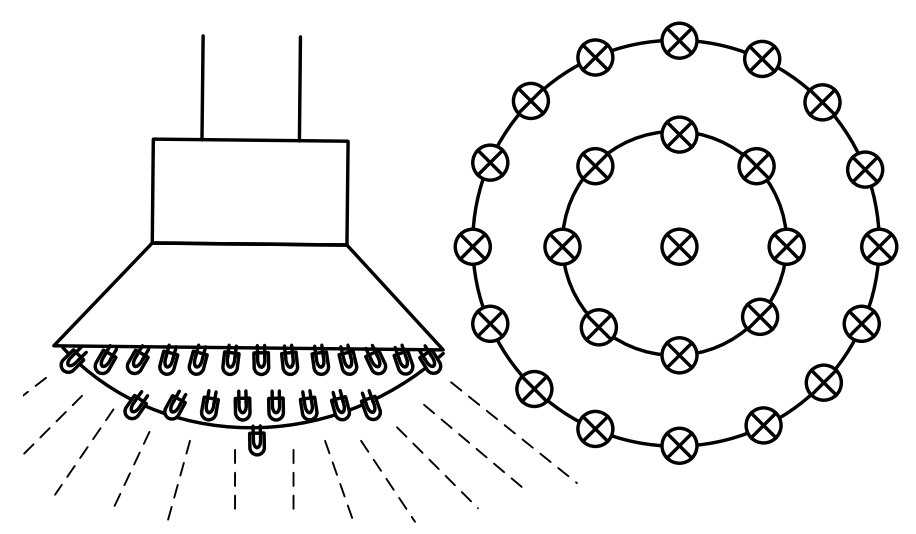

(a)

(b)

Figure 2.3: (a) 25-LED lamp structure from the side view, (b) 25-LED lamp structure from the bottom view

\subsection{Indoor VLC Channel Model}

In this section, we analyze the indoor VLC channel model and show the derivation of the channel model with the 1-LED and 25-LED lamp models.

For indoor VLC systems, white LEDs work as transmitters and photo-detectors work as receivers. Since the visible light is incoherent, intensity modulation and direct detection are employed in VLC systems. After the receiver, the received signal can be represented as

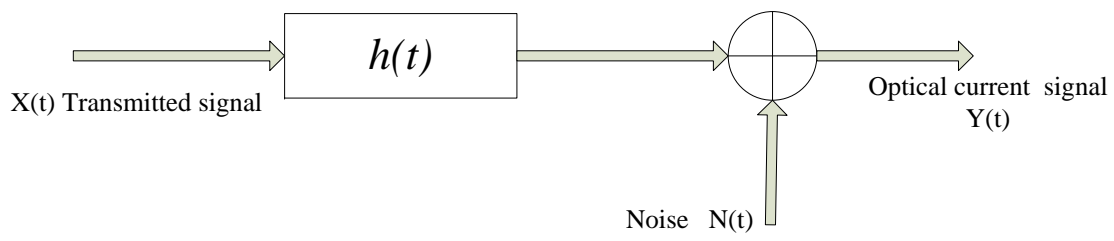

Figure 2.4: Basic indoor VLC channel model

$$
Y(t)=r X(t) \otimes h(t)+N(t)
$$

where $r$ represents the responsivity, $\otimes$ is convolution, $X(t)$ is the transmitted optical intensity, and $N(t)$ is the additive noise. $h(t)$ is the indoor channel impulse response, 
which, using ray-tracing, can be modeled as

$$
h(t)=\sum_{n=1}^{N} a_{n} \delta\left(t-t_{n}\right)
$$

where $a_{n}$ and $t_{n}$ represent the path gain and transmission time delay, respectively. $N$ is the number of multi-path components. The channel gains and transmission time delays in (2.2) depend on the light paths to the receiver.

Because of the principles of optics, the light rays from the transmitter can be classified into two types. They are the line of sight (LOS) rays and diffused rays, as shown in Figure 2.5. These two types cause the multi-path effect in indoor VLC system. Thus, the indoor VLC channel transfer function can be approximated by [1]

$$
H(f)=H_{L O S}(f)+H_{\text {diff }}(f)
$$

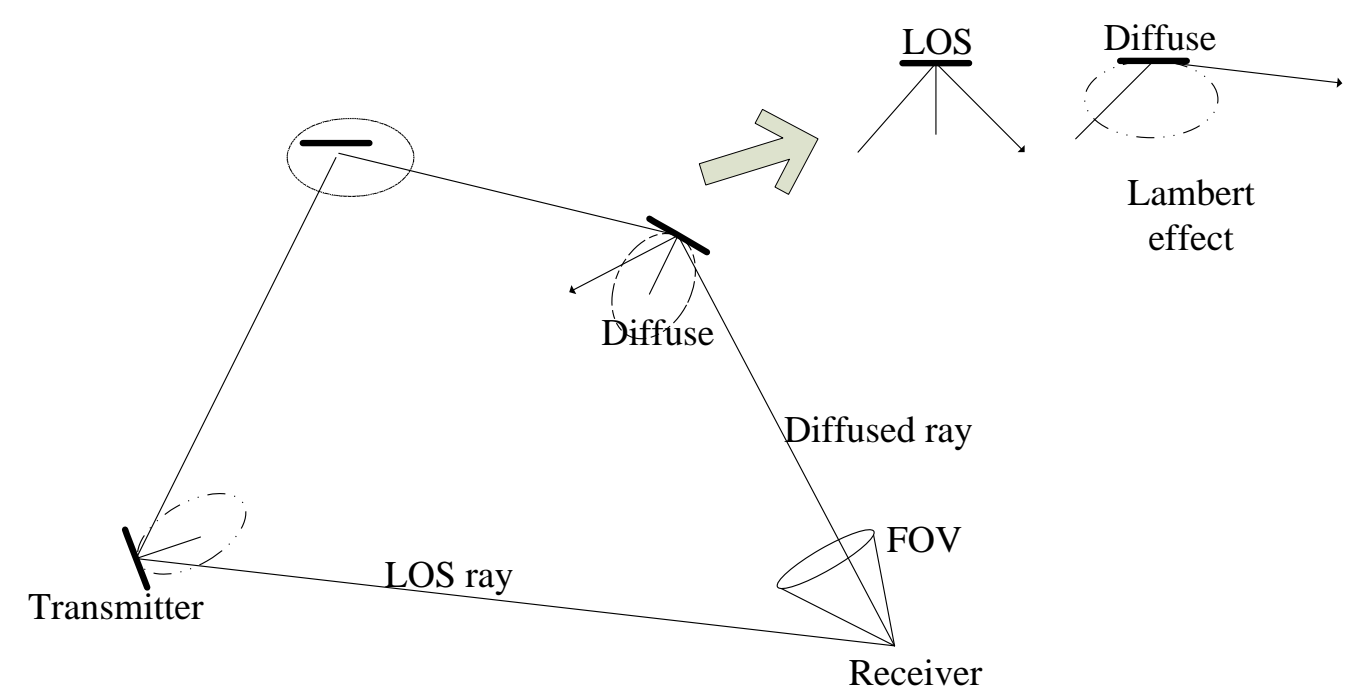

Figure 2.5: Light rays classification

where $H_{L O S}(f)$ is the contribution due to the LOS, which is basically independent of the modulation frequency, and it depends on the distance between transmitter and receiver and on their orientation with respect to the LOS. $H_{\text {diff }}(f)$ is the diffused 
part, the intensity of which is less than the LOS part. The impulse response of the indoor channel can also be represented as

$$
\begin{aligned}
h(t) & =\sum_{k=0}^{\infty} h^{(k)}(t) \\
& =\underbrace{h^{(0)}(t)}_{\text {LOS part }}+\underbrace{\sum_{k=1}^{\infty} h^{(k)}(t)}_{\text {Diffused part }},
\end{aligned}
$$

where $k$ is the number of reflections.

The intensity of the LOS rays and diffused rays follow the Lambertian law. The Lambertian radiant intensity model can be defined as [15]

$$
R_{0}(\phi)= \begin{cases}\frac{m+1}{2 \pi} \cos ^{m}(\phi) & \text { for } \phi \in[-\pi / 2, \pi / 2] \\ 0 & \text { for }|\phi| \geq \pi / 2\end{cases}
$$

where $m$ is the Lambertian mode of the light source and $\phi$ is the radiation angle for transmitters as shown in Figure 2.6. The maximum radiated power is reached when $\phi=0$. The Lambertian mode $m$ is related to the LED's semiangle $\Phi_{1 / 2}$ by

$$
m=\frac{\ln 2}{\ln \left(\cos \Phi_{1 / 2}\right)} .
$$

The detector effective area can be modelled as a function of the incident angle, $\psi$, as $[15]$

$$
A_{\text {eff }}(\psi)=\left\{\begin{array}{ll}
A_{r} \cos \psi & -\pi / 2 \leq \psi \leq \pi / 2 \\
0 & |\psi|>\pi / 2
\end{array},\right.
$$

where $A_{r}$ is the area of the detector as shown in Figure 2.6. We assume that the detector cannot be active beyond the field of view (FOV) angle $\Psi_{c}$. Therefore, the 


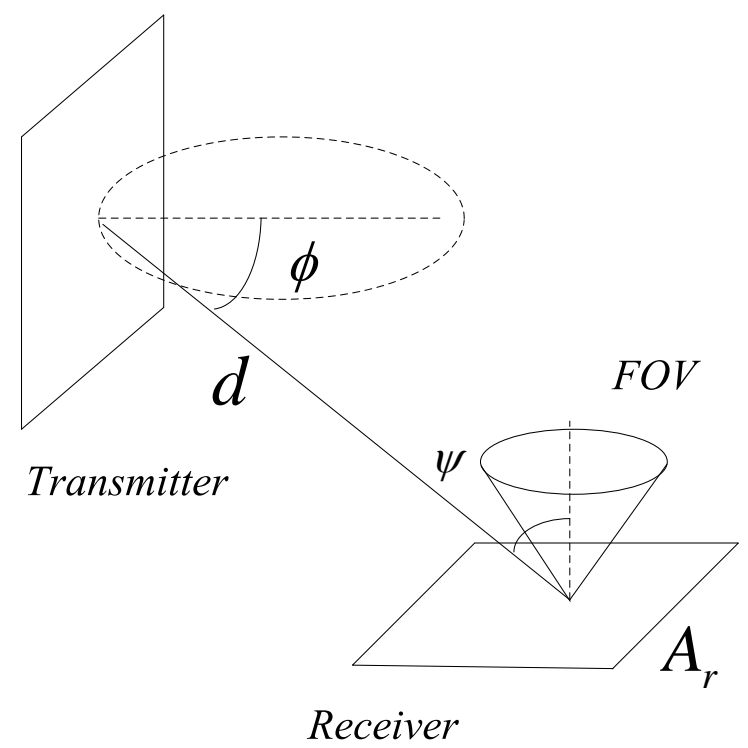

Figure 2.6: LOS light rays model

LOS link can be described as

$$
H_{L O S}=\left\{\begin{array}{ll}
\frac{A_{r}(m+1)}{2 \pi d^{2}} \cos ^{m}(\phi) \cos (\psi) & -\Psi_{c} \leq \psi \leq \Psi_{c} \\
0 & \text { elsewhere }
\end{array} .\right.
$$

Thus, the impulse response of LOS part can be described as

$$
h^{(0)}(t)=h_{L O S}(t)=\frac{A_{r}(m+1)}{2 \pi d^{2}} \cos ^{m}(\phi) \cos (\psi) \delta\left(t-\frac{d}{c}\right)
$$

The diffused part is

$$
h^{(k)}(t)=A_{r} L_{0} L_{1} L_{2} \ldots L_{k} \gamma^{k} \delta\left(t-\frac{d_{0}+d_{2}+\ldots d_{k}}{c}\right)
$$

where $L_{0}, L_{1}, \ldots, L_{k}$ represent the link attenuations, $\gamma$ is the reflection coefficient, 


$$
\begin{aligned}
L_{0} & =\frac{(m+1) \cos ^{m}\left(\phi_{0}\right) \cos \left(\psi_{0}\right)}{2 \pi d_{0}^{2}} \\
L_{1} & =\frac{\cos ^{m}\left(\phi_{1}\right) \cos \left(\psi_{1}\right)}{\pi d_{1}^{2}} \\
\vdots & \\
L_{k} & =\frac{\cos ^{m}\left(\phi_{k}\right) \cos \left(\psi_{k}\right)}{\pi d_{k}^{2}}
\end{aligned}
$$

where $d_{0}$ is the distance of the LOS link, $d_{k}$ represents the distance of the $k$ th bounce link, $\phi$ is the radiation angle, $\psi$ represents the incident angle, $d$ and $c$ represent distance between transmitter and receiver and light speed, respectively [25].

\subsection{Numerical Results of the Indoor Channel}

In this section, we use a ray-tracing method to calculate the channel gains and impulse response of the channel. We assume that one illumination point represents $0.01 \mathrm{~m}^{2}$, therefore there are 2500 points on the floor and 1500 points on each wall of a $5 \mathrm{~m} \times 5 \mathrm{~m} \times 3 \mathrm{~m}$ room. To calculate the diffused part of the impulse response of the channel, we make the light rays reach every point on the four walls, and then these light rays are diffused to the receiver (single bounce). After summing up all the light rays diffused from the four walls with time delay, we can get the diffused part of the impulse response. For the LOS part, it is easy to sum up all the light rays received by the receiver directly.

To analyze the characteristics of the indoor VLC channel and transmitter models, numerical results are shown in this section. The performance of the 1-LED and 25LED lamp models are compared, and the power distributions with different LED semiangle are analyzed.

Before finding numerical results, we rewrite the general formula of the LOS link 


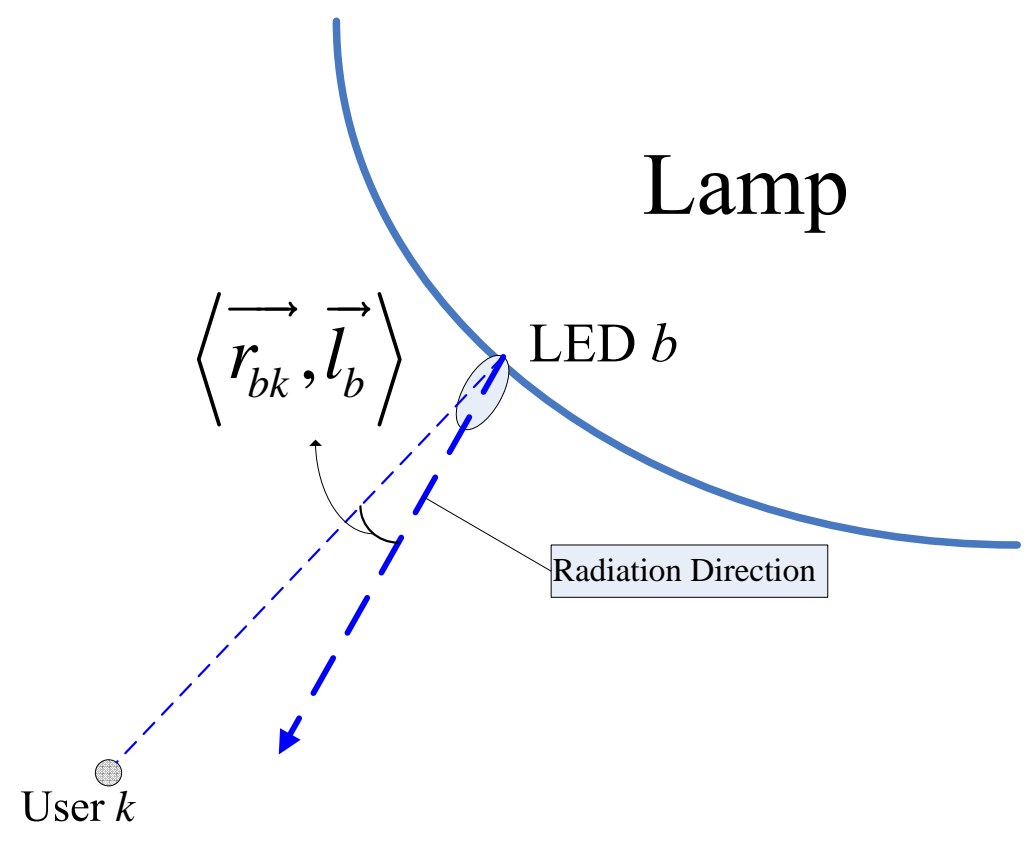

Figure 2.7: Angle between radiation direction and user

between the $i$ th LED and the $k$ th PD as

$$
H_{i k}=\frac{A_{r} \cos \left\langle\overrightarrow{r_{i k}}, \overrightarrow{n_{k}}\right\rangle}{2 \pi d_{i k}^{2}}(m+1) \cos ^{m}\left\langle\overrightarrow{r_{i k}}, \overrightarrow{l_{i}}\right\rangle
$$

where $d_{i k}$ is the distance between the $i$ th LED and the $k$ th user. $\overrightarrow{r_{i k}}$ is the unit vector from $i$ th LED to the $k$ th user, $\overrightarrow{n_{k}}$ is the $k$ th receiver's normal unit vector, and $\overrightarrow{l_{i}}$ represents the radiation unit direction vector for the $i$ th LED. In $(2.12),\langle x, y\rangle$ represents the angle between vectors $x$ and $y$. Figured 2.7 shows $\left\langle\overrightarrow{r_{i k}}, \overrightarrow{l_{i}}\right\rangle$, the angle between the direction of LED $i$ and its LOS path to user $k$.

From the general formula of the channel, the semiangle of the LED effects the power distributions on the floor. The power distributions of different semiangles from $3^{\circ}$ to $40^{\circ}$ are shown in this section. It is assumed that our indoor environment is a $5 \mathrm{~m} \times 5 \mathrm{~m} \times 3 \mathrm{~m}$ empty room as shown in Figure 2.8. There are four lamps on the ceiling at positions $(1.25,1.25,3),(1,25,3,75,3),(3,75,1,25,3),(3,75,3,75,3)$, in meters measured from one lower corner of the room. The reflection coefficient used 
is $\gamma=0.8$. For each LED lamp, the total radiation power is $300 \mathrm{~mW}$.

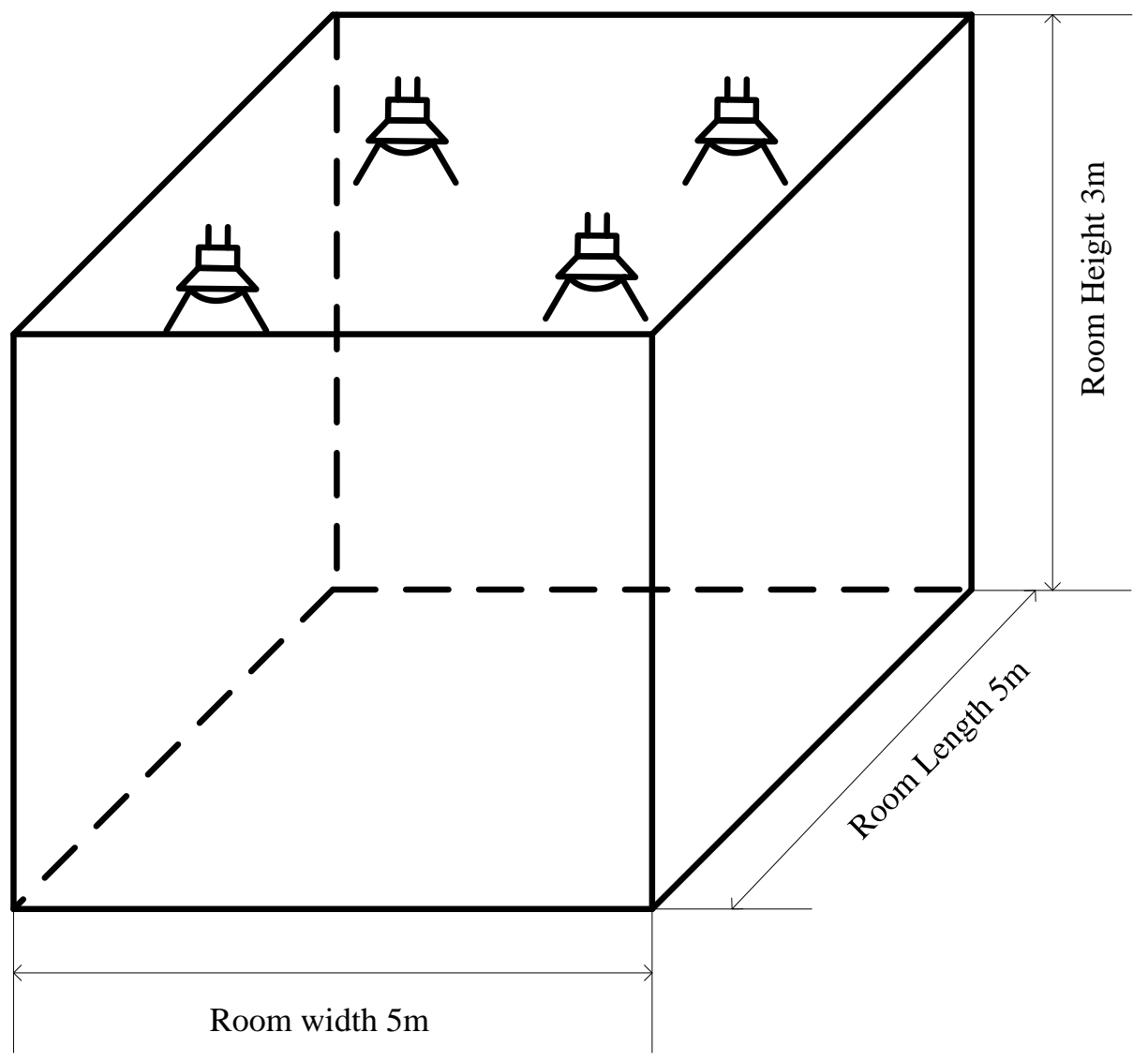

Figure 2.8: Indoor environment

Since the illuminance is usually represented in lux, we show the illumination distributions in lux with different semiangles in Figures 2.9-2.11 for the 1-LED lamp model. The standard office illuminance determined by the Illuminating Engineering Society of North America at a distance of $2.2 \mathrm{~m}$ from the ceiling is $400 \mathrm{~lx}$ [23]. Comparing these results, the power distributions with a $10^{\circ}$ semiangle has the highest illumination level, but has the smallest illumination coverage area. Therefore, it cannot satisfy the illumination requirement for the users who are not located right under the LEDs, as shown in Figure 2.9. Considering both the illumination coverage area and the illumination level, the cases of $20^{\circ}$ and $30^{\circ}$ semiangles are the reasonable light source candidates for indoor VLC systems. 


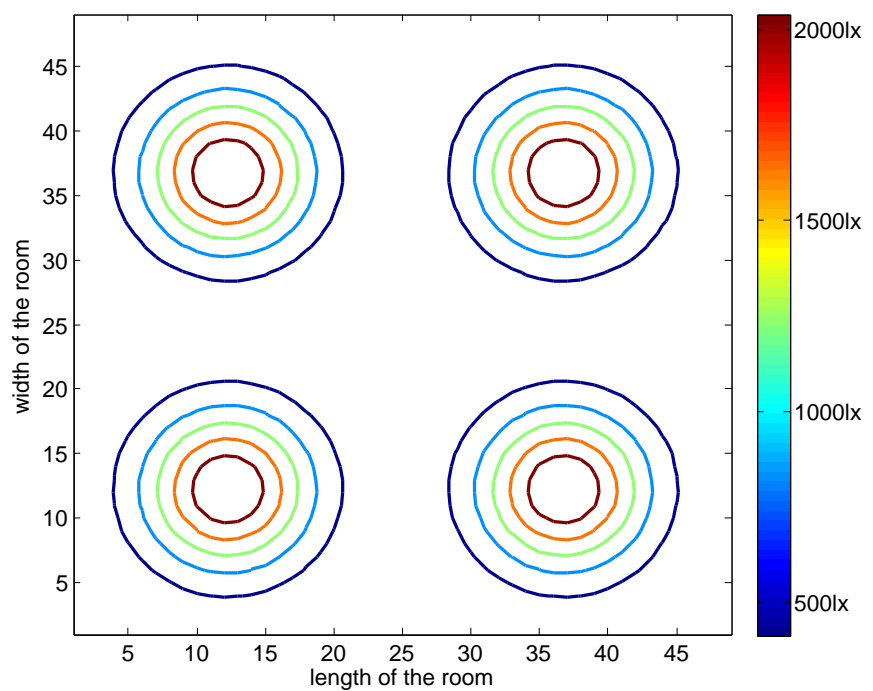

Figure 2.9: Illuminance (LOS and diffuse) distribution of 1-LED lamp with semiangle $=10^{\circ}$, in lux

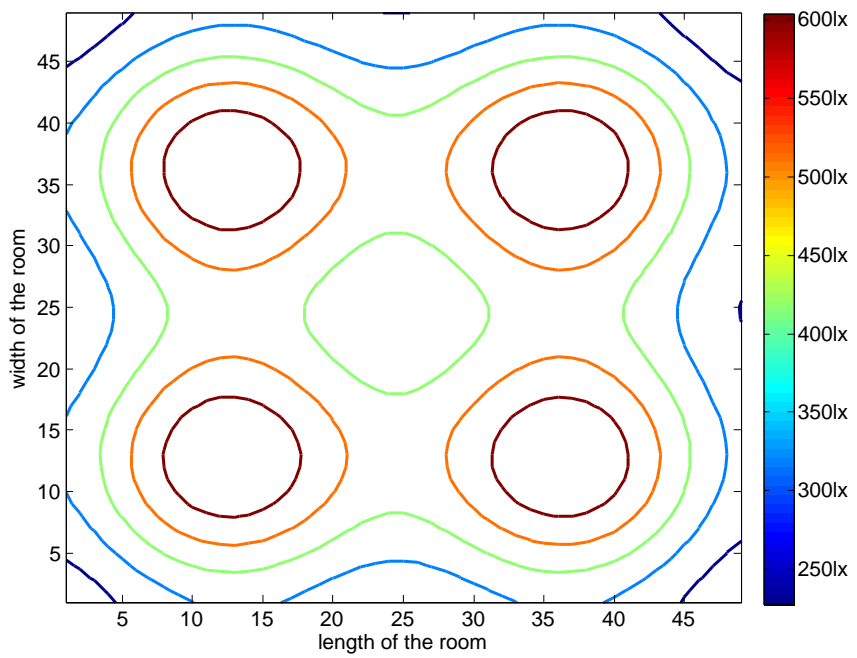

Figure 2.10: Illuminance (LOS and diffuse) distribution of 1-LED lamp with semiangle $=20^{\circ}$, in lux 


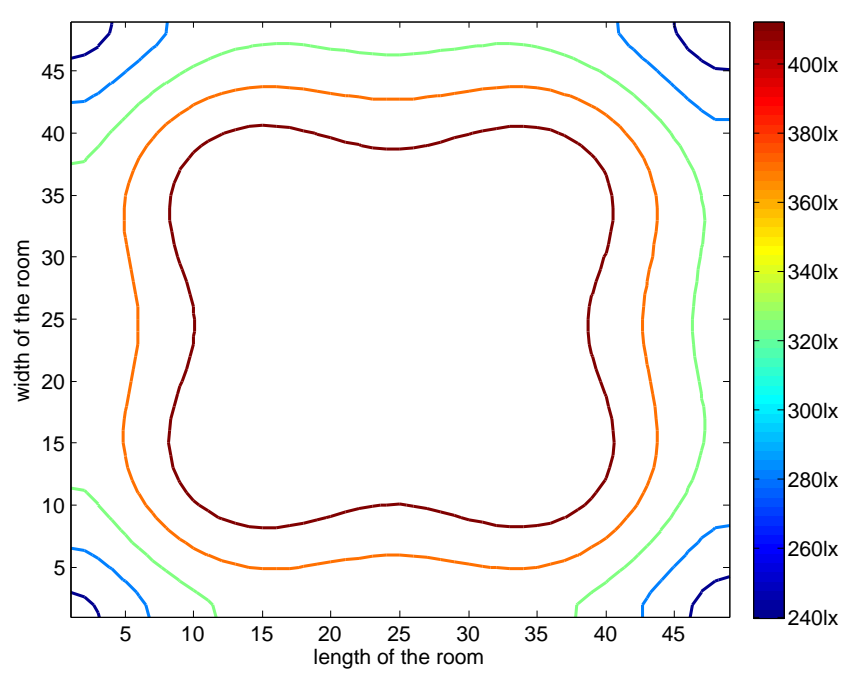

Figure 2.11: Illuminance (LOS and diffuse) distribution of 1-LED lamp with semiangle $=30^{\circ}$, in lux

For the 25-LED lamp model, we set up the semiangle for each LED in the 25LED lamp varying from $3^{\circ}$ to $40^{\circ}$. The illumination distributions are shown in Figures 2.12-2.16. According to these figures, the $3^{\circ}$ semiangle case has the highest intensity level but the smallest illumination coverage. Comparing the $20^{\circ}$ semiangle case of the 25-LED lamp in Figure 2.10 and the $30^{\circ}$ semiangle case of the 1-LED lamp in Figure 2.11, the illumination coverage area is similar but the the former has a higher illumination level. From these results, the 25-LED lamp model has an advantage over the 1-LED lamp model in illumination coverage with the same LED semiangle.

Besides the power distribution, the impulse response of the indoor channel can also be shown. It is assumed that the receiver is located at $(1.25,0.625,0)$ on the floor. The normalized impulse response of the $30^{\circ}$ in 1-LED case is shown in Figure 2.17. In this figure, LOS light makes the dominant contribution to the impulse response of the channel compared with the diffused light. From the impulse response, if the symbol rate is lower than 200 Msymbols/s approximately, the multi-path effect can be neglected.

For the 25-LED case, the normalized impulse response with a $30^{\circ}$ semiangle is 


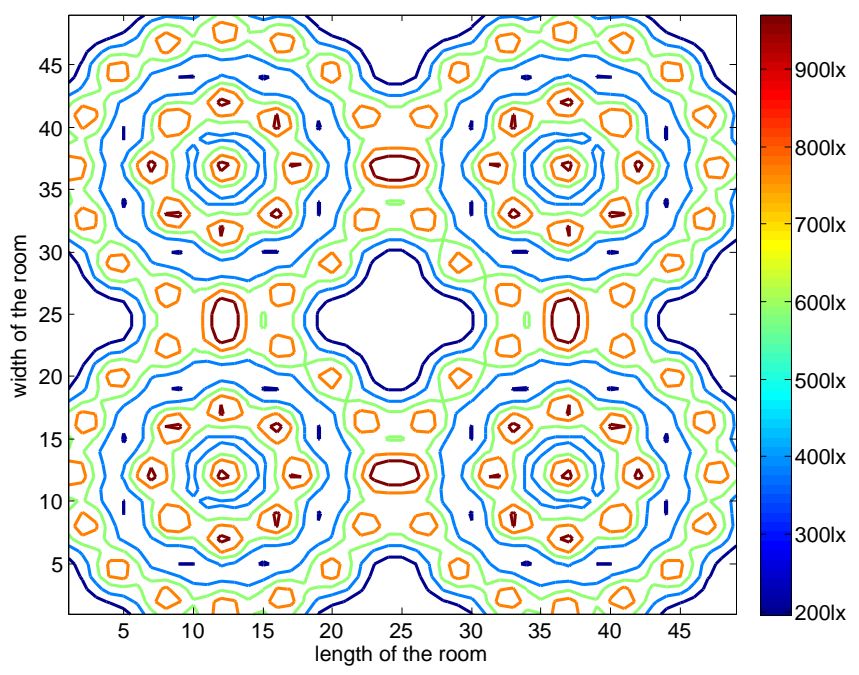

Figure 2.12: Illuminance (LOS and diffuse) distribution of 25-LED lamp with semiangle $=3^{\circ}$, in lux

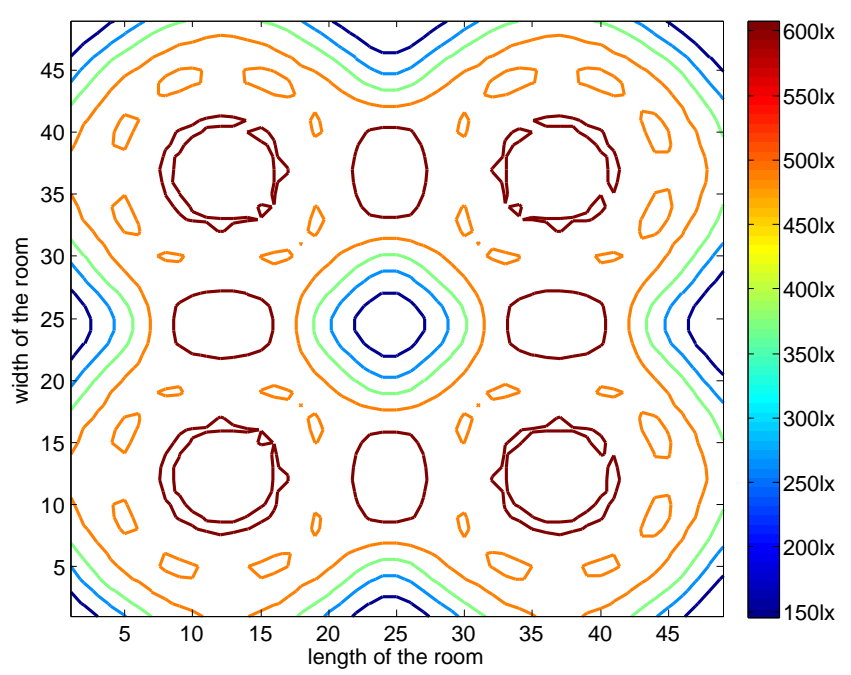

Figure 2.13: Illuminance (LOS and diffuse) distribution of 25-LED lamp with semiangle $=5^{\circ}$, in lux 


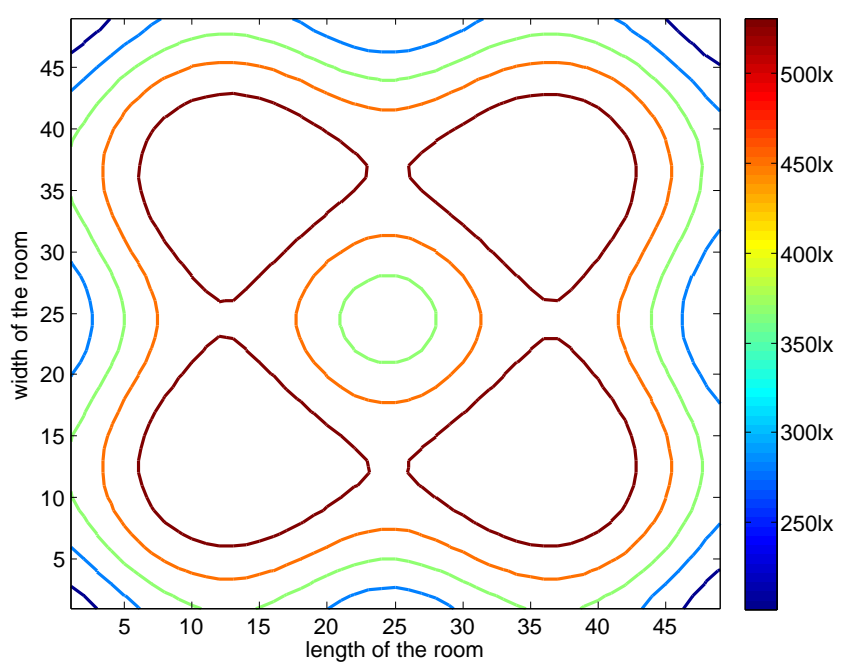

Figure 2.14: Illuminance (LOS and diffuse) distribution of 25-LED lamp with semiangle $=10^{\circ}$, in lux

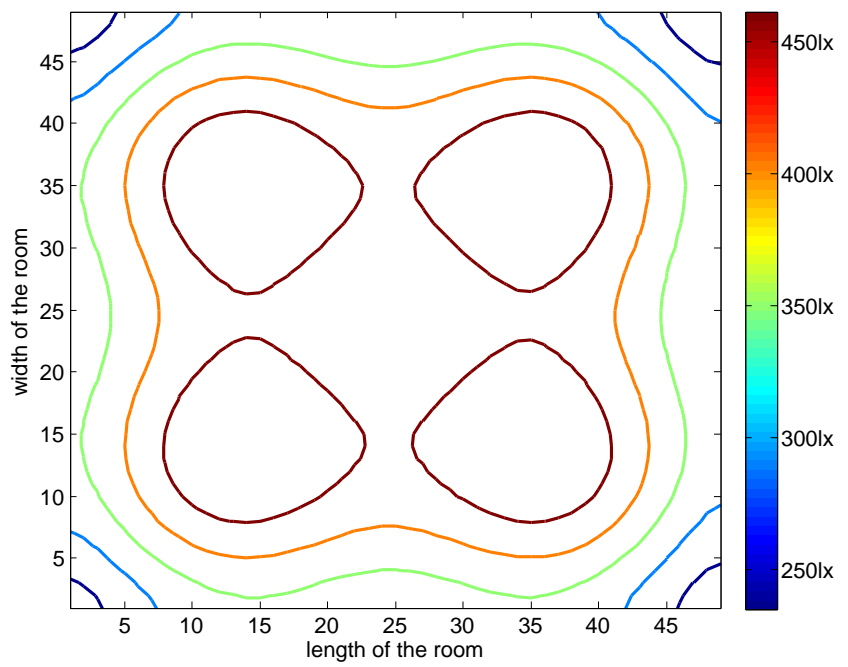

Figure 2.15: Illuminance (LOS and diffuse) distribution of 25-LED lamp with semiangle $=20^{\circ}$, in lux 


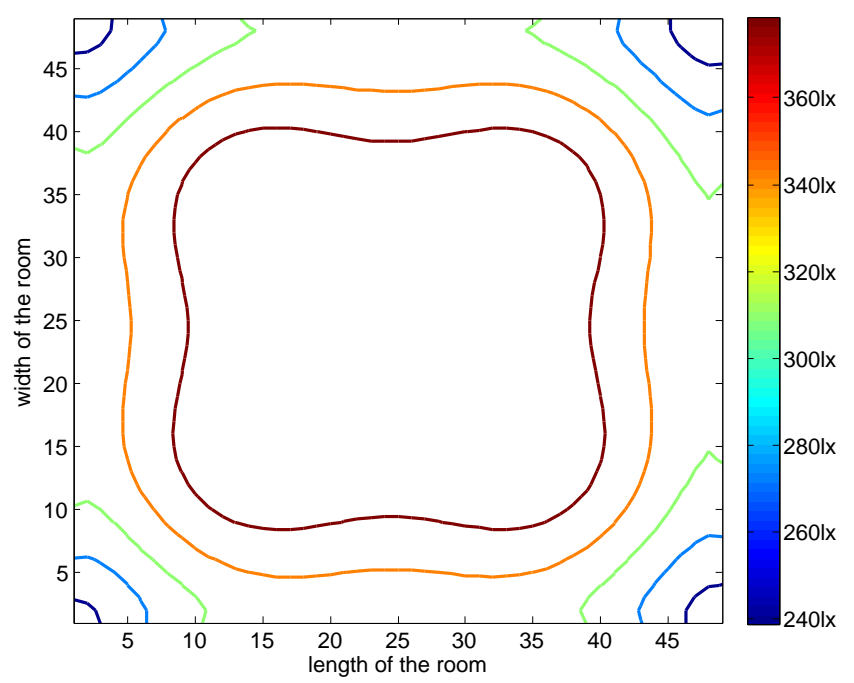

Figure 2.16: Illuminance (LOS and diffuse) distribution of 25-LED lamp with semiangle $=30^{\circ}$, in lux

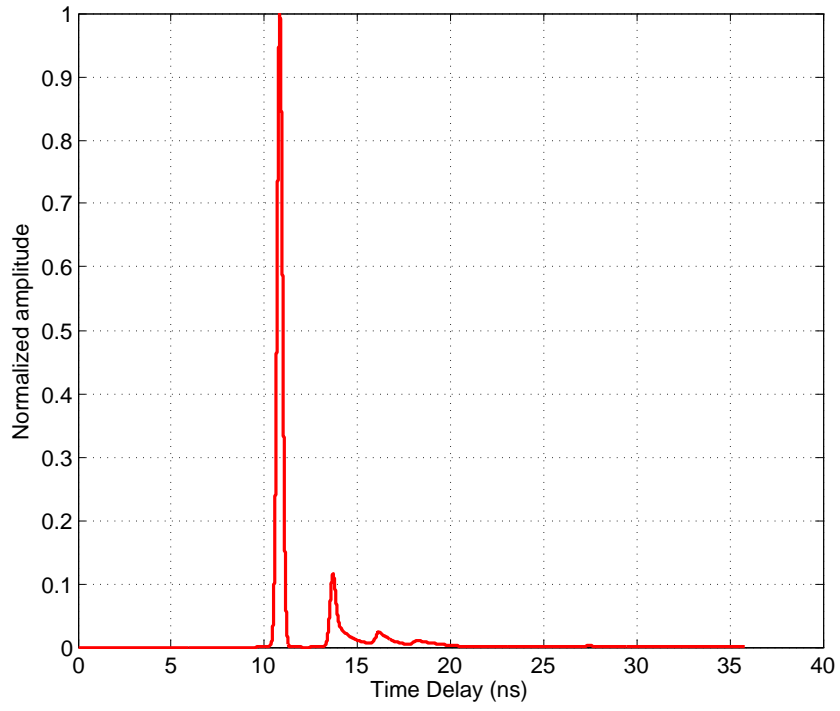

Figure 2.17: Normalized impulse response of 1-LED lamp with semiangle $=30^{\circ}$ at $(1.25,0.625,0)$ 


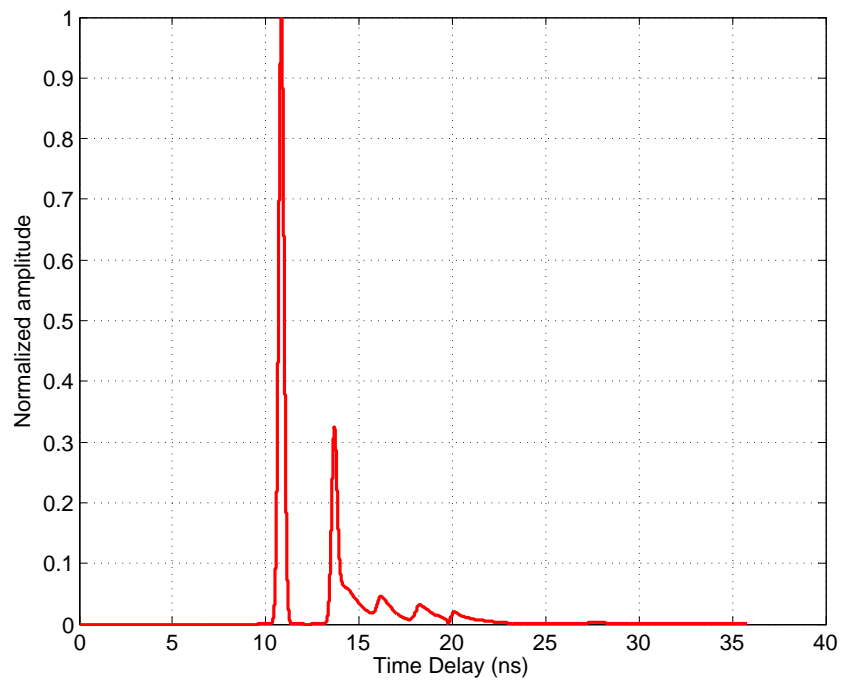

Figure 2.18: Normalized impulse response of 25-LED lamp with semiangle $=30^{\circ}$ at $(1.25,0.625,0)$

shown in Figure 2.18. Comparing this figure to the 1-LED case, since the 25-LED model covers a larger illumination area, more light is diffused from the walls to the receiver. Therefore, the multi-path effect is much stronger than in the 1-LED case. 


\section{Chapter 3}

\section{One User VLC System}

In this chapter, we analyze the performance of the one user case in indoor VLC systems. Since the visible light is incoherent, intensity modulation and direct detection are employed. Considering the intensity modulation scheme, M-ary pulse amplitude modulation (M-PAM) has been widely used in optical communication area because of its simple transmitter and receiver structure. For the one user case, on-off keying (2-PAM) and M-PAM scheme are employed in this chapter to transmit the signals.

\subsection{Signal to Noise Ratio and Bit Error Rate Anal- ysis for On-Off Keying}

When the visible light is received by the detector, the optical power is converted into electrical power. The electrical signal to noise ratio (SNR) is described as

$$
S N R=\frac{\left(r P_{r}\right)^{2}}{\sigma_{\text {shot }}^{2}+\sigma_{\text {background }}^{2}+\sigma_{\text {thermal }}^{2}},
$$


where, $r$ is the photodetector responsivity. Usually, $r$ should be around $0.6 \mathrm{~A} / \mathrm{W}$ to $0.8 \mathrm{~A} / \mathrm{W} . P_{r}$ is the optical power received by the detector, $\sigma_{\text {shot }}^{2}, \sigma_{\text {background }}^{2}$ and $\sigma_{\text {thermal }}^{2}$ represent shot noise variance, background light noise variance and thermal noise variance. Here, the shot noise variance, background light noise variance and thermal noise variance are defined as [7]

$$
\begin{gathered}
\sigma_{\text {shot }}^{2}=2 q r P_{r} B, \\
\sigma_{\text {background }}^{2}=2 q I_{b} I_{2} B, \\
\sigma_{\text {thermal }}^{2}=4 \kappa T_{\kappa} B / R_{L},
\end{gathered}
$$

where $q$ is the electronic charge, $B$ is equivalent to the noise bandwidth, $\kappa$ is the Boltzmann's constant, $I_{b}$ is the background light current, $I_{2}$ is the bandwidth factor, $T_{\kappa}$ is the absolute temperature, and $R_{L}$ is the resistor in the circuit of the receiver.

In VLC systems, though the transmitted signal for the on-off keying (OOK) scheme is "1" or "0" absolutely, the received signal is not, due to the shot noise, background noise and thermal noise. When sending "1", if the amplitude of the received signal is less than the threshold $D$, the received signal would be decided as "0". On the contrary, there is some probabilities that the transmitted "0" would be decided as "1" if the received power is higher than the threshold because of the noise.

For the VLC receiver, every received bit contains a large numbers of photons, thus, the Poisson distribution for the shot noise can be regarded as a Gaussian distribution, approximately. Let $S_{1}$ and $S_{0}$ represent the average signal after the receiver. Thus, the probability for sending " 1 " and deciding " 0 " is

$$
P(0 \mid 1)=\frac{1}{2} \operatorname{erfc}\left(\frac{S_{1}-D}{\sigma_{1} \sqrt{2}}\right) .
$$


On the contrary, the probability for sending "0" and deciding "1" is

$$
P(1 \mid 0)=\frac{1}{2} \operatorname{erfc}\left(\frac{D-S_{0}}{\sigma_{0} \sqrt{2}}\right)
$$

where $\sigma_{1}^{2}$ and $\sigma_{0}^{2}$ are the noise variances for sending "1" and "0", which can be described as

$$
\begin{aligned}
& \sigma_{0}^{2}=2 q r P_{r 0} B+\sigma_{\text {background }}^{2}+\sigma_{\text {thermal }}^{2}, \\
& \sigma_{1}^{2}=2 q r P_{r 1} B+\sigma_{\text {background }}^{2}+\sigma_{\text {thermal }}^{2}
\end{aligned}
$$

where $P_{r 0}$ represents the received power when sending $0, P_{r 1}$ is the received power when sending 1 . Therefore, if we assume that the probability for sending "1" and "0" are given as $P(1)$ and $P(0)$, respectively, the bit error rate (BER) can be described as

$$
\mathrm{BER}=P(1) P(0 \mid 1)+P(0) P(1 \mid 0) .
$$

Substituting (3.5) and (3.6) into (3.8), if $P(0)=P(1)$ we can find

$$
\mathrm{BER}=\frac{1}{4}\left[\operatorname{erfc}\left(\frac{S_{1}-D}{\sigma_{1} \sqrt{2}}\right)+\operatorname{erfc}\left(\frac{D-S_{0}}{\sigma_{0} \sqrt{2}}\right)\right],
$$

where $\operatorname{erfc}(\cdot)$ is the complementary error function, which is defined as

$$
\operatorname{erfc}(x)=\frac{2}{\sqrt{\pi}} \int_{x}^{\infty} e^{-u^{2}} d u
$$

To analyze the noise in this system, we assume the parameters as shown in Table 3.1. The photo detector we select can be found in [27]. From this table, the thermal noise is the dominant noise in our system. Therefore $\sigma_{0}^{2}$ and $\sigma_{1}^{2}$ can be assumed to be approximately the same [5]. 
Table 3.1: Parameters and Estimates of Noise Variance

\begin{tabular}{cc}
\hline received power & $0.04 \mathrm{~mW} / \mathrm{cm}^{2}(400 \mathrm{~lx})$ \\
symbol rate & $70 \mathrm{M} / \mathrm{s}$ \\
photodetector responsivity & $0.6 \mathrm{~A} / \mathrm{W}$ \\
absolute temperature & $298 \mathrm{~K}$ \\
background light current & $1.38 \times 10^{-5} \mathrm{~A}$ \\
photon detector area & $0.3 \mathrm{~cm}^{2}$ \\
bandwidth factor $I_{2}$ & 0.52 \\
capacitance of this detector & $110 \mathrm{pF}$ \\
resistor in the circuit of the receiver & $36 \Omega$ \\
\hline$\sigma_{\text {shot }}^{2}$ & $1.61 \times 10^{-16}$ \\
$\sigma_{\text {background }}^{2}$ & $1.61 \times 10^{-16}$ \\
$\sigma_{\text {thermal }}^{2}$ & $5.56 \times 10^{-14}$ \\
\hline
\end{tabular}

\subsection{M-PAM Modulation Scheme}

The conventional modulation schemes adopted in RF communications cannot be readily applied in VLC directly, because the visible light is incoherent. Intensity modulation and direct detection are used, so non-negative signals are transmitted. Thus we need specific modulation schemes for optical communications. Pulse amplitude modulation is a spectrally efficient modulation scheme suitable for optical system. We use it in this chapter to support one user in an indoor VLC system.

An example for 4-PAM modulation is given in Figure 3.1 to help us understand the principle of the M-PAM scheme. The data stream ready for transmission is "000111100111" in this figure. For 4-PAM, we divide the stream into groups con- 
taining 2 bits. Here, the stream can be divided into " $00 "$, " $01 "$, " $11 "$, " $10 "$, " $01 "$, "11". Converting these binary numbers into 4-ary ones, we get $0,1,3,2,1,3$ for each group. For 4-PAM, the transmitters just need to send the corresponding power levels.

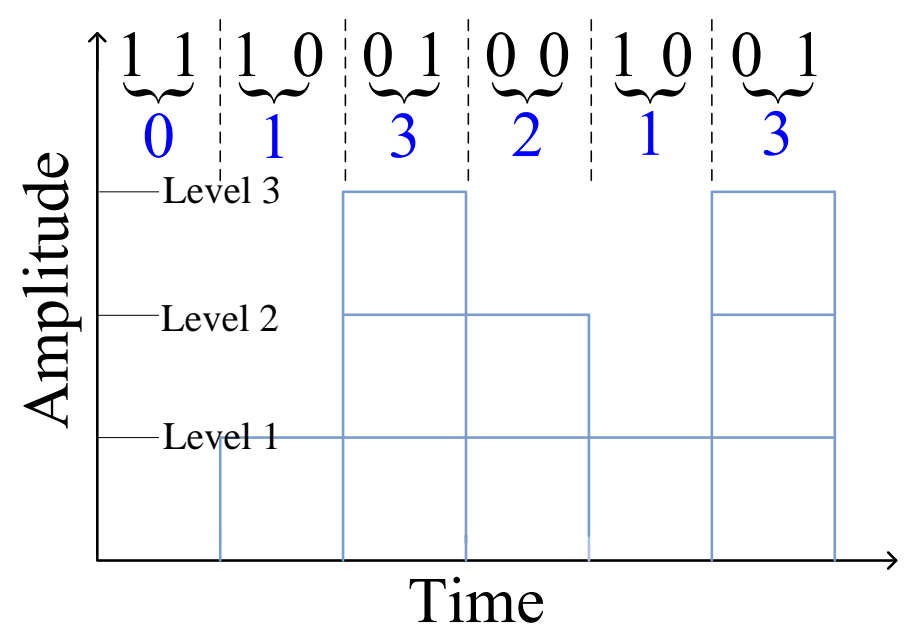

Figure 3.1: 4-PAM modulation

For an M-PAM modulation scheme, we suppose $A_{n} \in\left\{a_{0}, a_{1}, \ldots a_{M-1}\right\}$, where $a_{0}, a_{1}, \ldots a_{M-1}$ represent the $M$ optical power levels. Thus, after M-PAM modulation, the signal could be represented as

$$
X(t)=\sum_{n=0}^{\infty} A_{n} G\left(t-T_{c}\right),
$$

where $G(t)$ is the rectangular function which is described as

$$
G(t)= \begin{cases}1 & 0<t \leq T_{c} \\ 0 & \text { otherwise }\end{cases}
$$

Therefore, the block diagram of M-PAM scheme can be shown in Figure 3.2. In this block diagram, $n(t)$ represents the noise, $y(t)$ is the received signal at the receiver. 


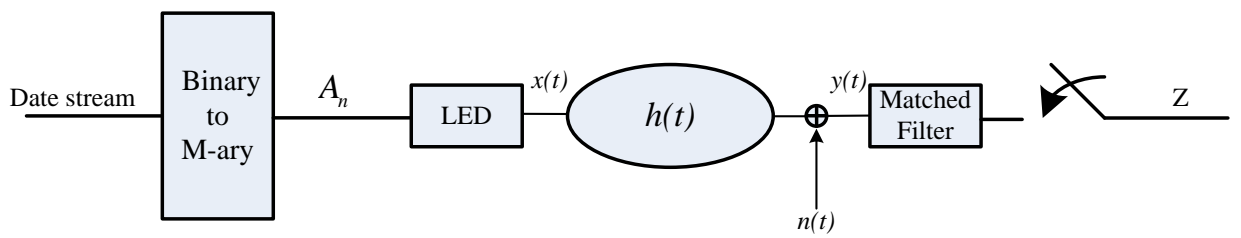

Figure 3.2: Block diagram of M-PAM schemes

The channel impulse response $h(t)$ is described in Chapter 2. After the matched filter and sampling, we can get the signal ready for demodulation and decision. Since we assume a rectangular pulse shape, for a sufficiently short channel response the matched filter is an integrate-and-dump filter.

To evaluate the performance of the M-PAM scheme, bandwidth efficiency and bit error rate (BER) are two significant criterion. Bandwidth efficiency refers to the information rate that can be transmitted over a given bandwidth in a specific communication system. It is a measure of how efficiently a limited frequency spectrum is utilized. M-PAM has a relative high bandwidth efficiency, which can be defined as

$$
\eta_{B}=\frac{R_{b}}{B_{t}}=\log _{2} M
$$

where $R_{b}$ is the achievable bit rate and $B_{t}$ is the bandwidth of the symbols.

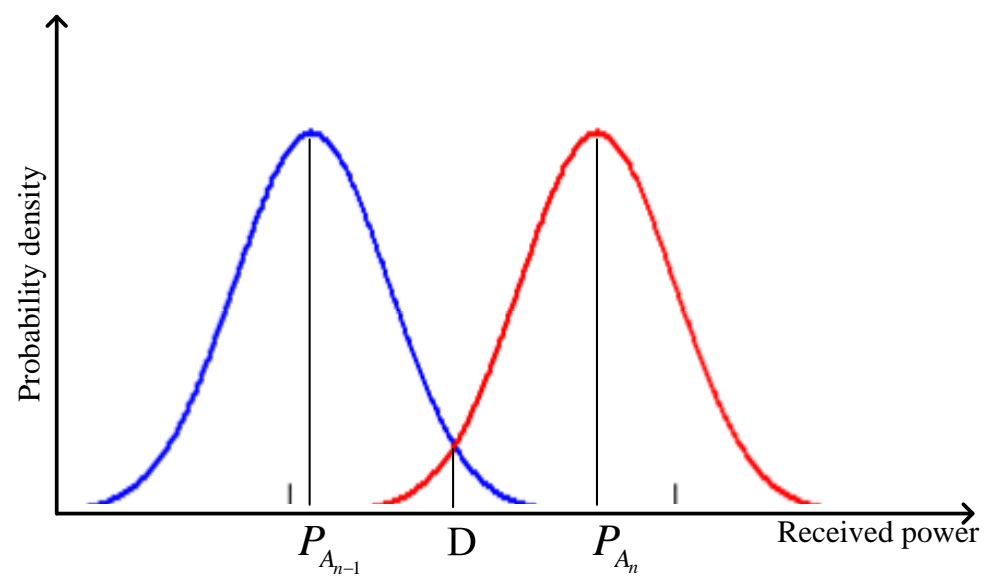

Figure 3.3: Optimal threshold with thermal noise 
Before we find the BER, the optimal threshold should be considered. Since the received power when sending $A_{n}$ and $A_{n-1}$ are $P_{A_{n-1}}$ and $P_{A_{n}}$, respectively, and the thermal noise is dominant, the optimal threshold $(D)$ is shown in Figure 3.3. In this figure, red and blue lines represent the received signal probability density distribution for the same noise variance. For the numerical results, we approximate the threshold and evaluate the BER using a Monte Carlo method.

For M-PAM scheme, the BER can be represented approximately as [15]

$$
P_{M-P A M}=\frac{(M-1)}{M \log _{2} M} \operatorname{erfc}\left(\sqrt{\frac{r^{2} P_{r}^{2}}{2 \sigma^{2}(M-1)^{2}}}\right)
$$

where $\sigma^{2}$ is the noise variance including shot noise, background noise and thermal noise. Since the thermal noise in this system is dominant, $\sigma^{2}$ can be described as

$$
\sigma^{2}=\sigma_{\text {shot }}^{2}+\sigma_{\text {background }}^{2}+\sigma_{\text {thermal }}^{2} \approx \sigma_{\text {thermal }}^{2}
$$

\subsection{Simulation Results of One User Case in Indoor VLC Systems}

For our indoor VLC system, the communication performance for the one user case depends on the user's location, LED's semiangle (beamwidth) and the modulation scheme. In this section, the BER distributions for a 4-PAM scheme with $5^{\circ}, 10^{\circ}$ and $20^{\circ}$ LED semiangles are shown. In addition, the one user BER performances using 2-PAM, 4-PAM and 8-PAM are analyzed.

The BER distributions with $5^{\circ}, 10^{\circ}$ and $20^{\circ}$ LED semiangles show us the relationship between the BER performance and the user's location with different LED semiangles, from Figures 3.4 to 3.6. According to the simulation results, the BER 


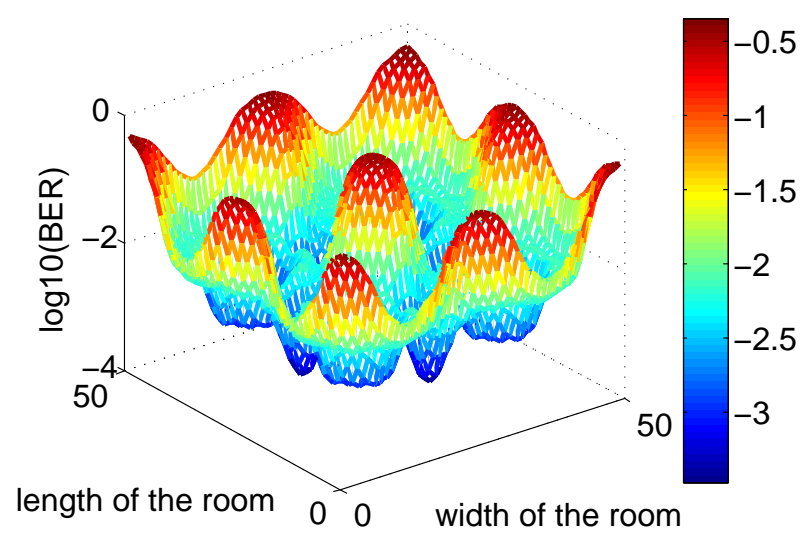

Figure 3.4: BER distribution of 4-PAM modulation using the 25-LED lamp with semiangle $=5^{\circ}$

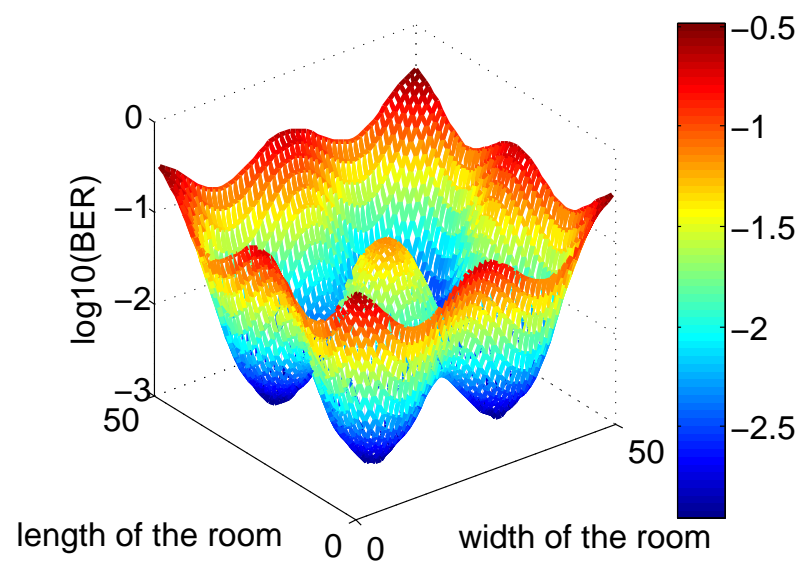

Figure 3.5: BER distribution of 4-PAM modulation using the 25-LED lamp with semiangle $=10^{\circ}$

performance of the $5^{\circ}$ semiangle case is the worst in general. Comparing the BER performance around the corner areas in Figures 3.5 and 3.6, the $20^{\circ}$ semiangle case is better than the $10^{\circ}$ semiangle case, since the darkest area in the $20^{\circ}$ case has a better BER than the darkest area in the $10^{\circ}$ case, and the BER distribution is more smooth for the $20^{\circ}$ semiangle case. From this point of view, the $20^{\circ}$ semiangle case is the best one among these three cases.

We then analyze the performance of the one user case in our indoor VLC environment, using the 1-LED lamp model. To get a clear comparison, we assumed that the 


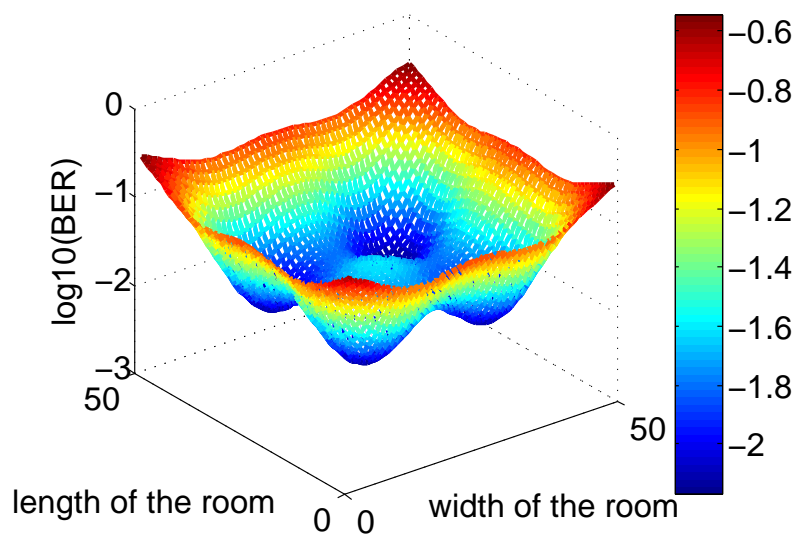

Figure 3.6: BER distribution of 4-PAM modulation using the 25-LED lamp with semiangle $=20^{\circ}$

user is located on the floor of the room at $(2.5,3.5,0)$. Theoretical BER performance (from (3.14)) and simulation BER performance are shown in Figure 3.7. According to the result, for a fixed symbol rate the 2-PAM modulation has the best performance in BER but the 8-PAM modulation has the largest bit transmission rate and bandwidth efficiency. Therefore, if the BER performance is of significant concern, the 2-PAM scheme should be the best choice. On the other hand, if bandwidth efficiency is what we care about, the 8-PAM scheme should be used. 


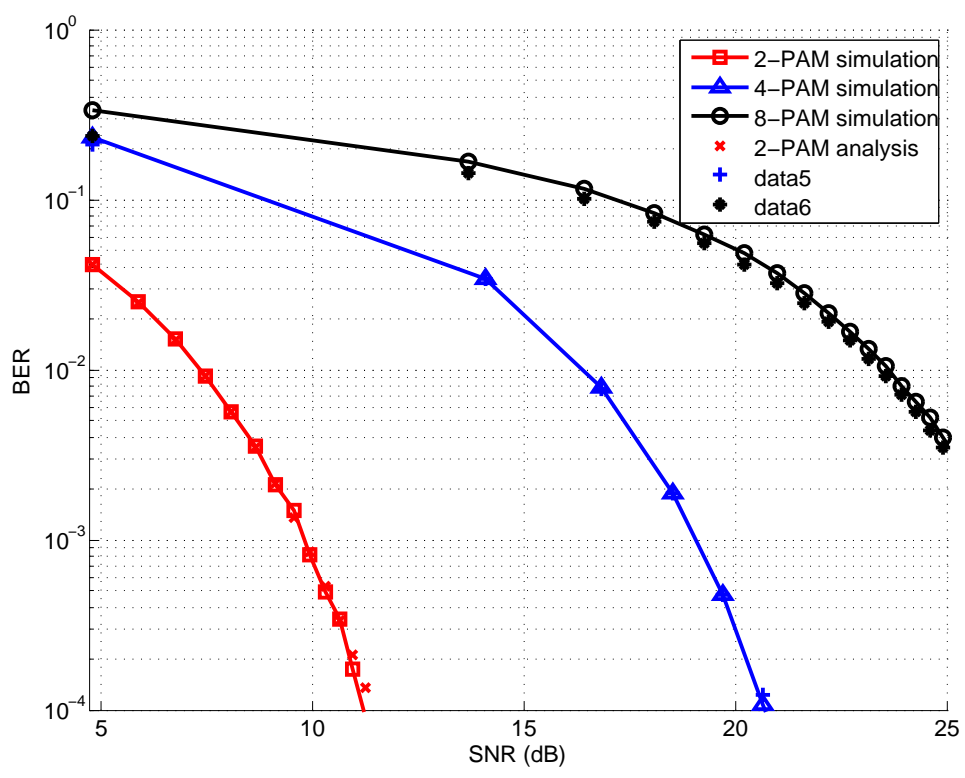

Figure 3.7: Theoretical BER performance and simulation BER performance of one user for 2-PAM, 4-PAM and 8-PAM for the 1-LED model 


\section{Chapter 4}

\section{Multiuser VLC System}

In this chapter, we use code division multiple access (CDMA) and multiple input single output (MISO) techniques to support multiple users in the indoor VLC system. To reduce the multiuser interference, a power allocation joint optimization (PAJO) algorithm is proposed in this chapter. Through the PAJO algorithm, the optimal power allocation maximizes the minimum signal to interference plus noise ratio (SINR) of all users and gives a minimum mean square error (MMSE) receivers design

scheme for different user. Furthermore, we compare the BER performance between the block diagonalization precoding algorithm proposed in [13] and PAJO. According to the simulation results, we conclude that PAJO has a better BER performance than the block diagonalization precoding algorithm. In this chapter, the illumination requirements for the indoor space is considered.

\subsection{Optical CDMA}

In our indoor VLC system, a CDMA technique is employed to provide multiple access for simultaneous users. Because of the intensity modulation used in VLC, the CDMA code is different in optical communication than in RF communication. 
To implement the CDMA technique in optical communications, choosing the proper optical CDMA (OCDMA) code is a significant step.

Nowadays, OCDMA is receiving increasing attention due to its enhanced information security. In an OCDMA system, different users share a common communication medium, thus multiple access is achieved by assigning OCDMA codes to different users [30]. Therefore, the transmitted signal for user $i$ can be described as $d_{i} \mathbf{c}_{i}$, where $d_{i}$ is the intended data for user $i$, and $\mathbf{c}_{i}=\left\{c_{i}[1], \ldots, c_{i}[L]\right\}$ represents the length- $L$ OCDMA code for user $i$.

An important type of OCDMA code is the optical orthogonal code (OOC) that was proposed for intensity modulation and direct-detection (IM-DD) optical CDMA (OCDMA) systems [24]. OCDMA codes must satisfy the following conditions [30]:

1. The peak auto-correlation function of the code should be maximized.

2. The cross-correlation between any codes should be minimized.

3. The side lobes of the auto-correlation function of the code should be minimized.

Conditions (1) and (2) insure that the multiple access interference (MAI) is minimized, and condition (3) insures the synchronization process at the receiver. In this thesis, since we consider only the downlink, the codes for all users are transmitted synchronously.

An OOC is usually represented by $\left(L, w, \lambda_{a}, \lambda_{c}\right)$, where $L$ is the code-length; $w$ is the code-weight; $\lambda_{a}$ is the upper-bound on the auto-correlation value for a non-zero shift and $\lambda_{c}$ is an upper-bound on cross-correlation values. The conditions for OOC are [30]

$$
R_{c_{i} c_{j}}[m]=\sum_{l=1}^{L} c_{i}[l] c_{j}[l+m] \leq \lambda_{c} \quad \forall m
$$


where $\mathbf{c}_{i}$ is the $i$ th codeword, and

$$
R_{c_{i} c_{i}}[m]=\sum_{l=1}^{L} c_{i}[l] c_{i}[l+m] \leq \lambda_{a} \quad \forall m \neq 0 .
$$

Table 4.1: OOC $(L, 3,1)$ sequence indexes for various length

\begin{tabular}{ll}
\hline$L$ & Sequence Index, When $L \leq 49, \lambda_{a}=\lambda_{c}=1$ \\
\hline 7 & $\{1,2,4\}$ \\
13 & $\{1,2,5\}\{1,3,8\}$ \\
19 & $\{1,2,6\}\{1,3,9\}\{1,4,11\}$ \\
25 & $\{1,2,7\}\{1,3,10\}\{1,4,12\}\{1,5,14\}$ \\
31 & $\{1,2,8\}\{1,3,12\}\{1,4,16\}\{1,5,15\}\{1,6,14\}$ \\
37 & $\{1,2,12\}\{1,3,10\}\{1,4,18\}\{1,5,13\}\{1,6,19\}\{1,7,13\}$ \\
43 & $\{1,2,20\}\{1,3,23\}\{1,4,16\}\{1,5,14\}\{1,6,17\}\{1,7,15\}\{1,8,19\}$ \\
\hline
\end{tabular}

There is a special case of $\lambda_{a}=\lambda_{c}=\lambda$ that the OOC is represented by $(L, w, \lambda)[30]$. Table 4.1 gives us examples for codeword indexes (the positions of the "ones" in the codeword). For example, the index $\{1,2,4\}$ with length 7 represents the codeword in Figure 4.1.

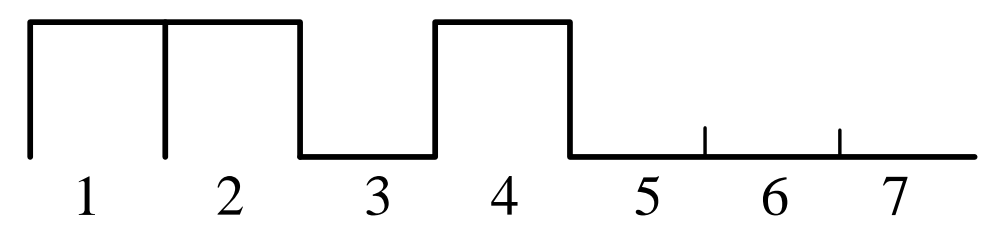

Figure 4.1: OOC $\{1,2,4\}$ with length of 7 


\subsection{VLC MISO Technology}

MIMO is an extension of MISO where each user is also equipped with multiple sensors. The original goal for MIMO and MISO is to improve the data transmission rate without the need for additional power or bandwidth. Although the MIMO technique has been employed to support simultaneous multiuser communication in RF communications for many years [17], MIMO in the optical communications area has not been shown to be an effective solution for multiuser networks until recent years [14]. In this chapter, MISO is employed to support multiple users to receive data simultaneously in indoor VLC. Each user employs a single photodetector. Figure 4.2 shows a block diagram of a typical indoor VLC MISO system. In this figure four LED lamps are used for room lighting as well as for transmitting independent data streams simultaneously [29].

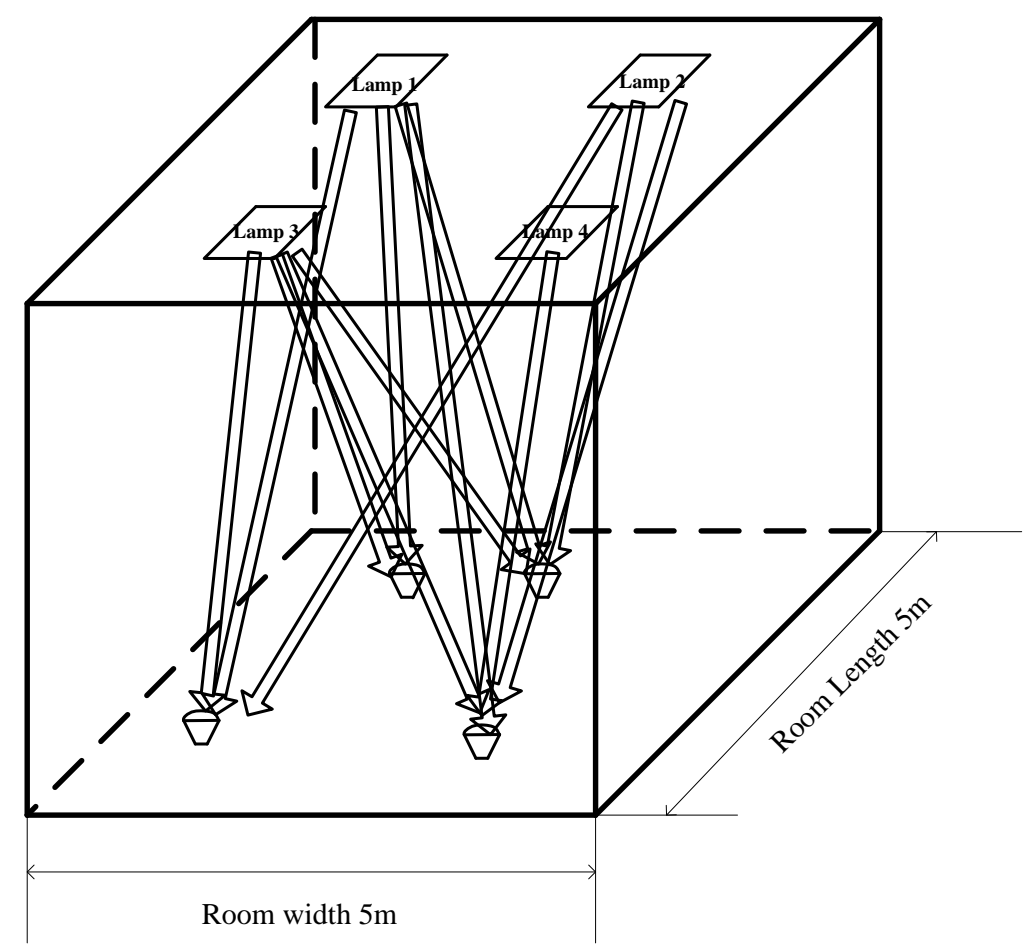

Figure 4.2: Block diagram of a typical VLC MISO system 
In a VLC MISO system, if the number of transmitters is $Q$ and the number of receivers is $K$, this MISO system channel can be represented as a $Q \times K$ matrix. In this thesis, we assume that there is no inter symbol interference (ISI), thus the MISO channel matrix can be described as

$$
\mathbf{H}=\left(\begin{array}{cccc}
h_{11} & h_{12} & \cdots & h_{1 K} \\
h_{21} & h_{22} & \cdots & h_{2 K} \\
\vdots & \vdots & \ddots & \vdots \\
h_{Q 1} & h_{Q 2} & \cdots & h_{Q K}
\end{array}\right)
$$

where $h_{i j}$ represents the channel attenuation from the $i$ th transmitter to the $j$ th receiver. For the indoor VLC system, $h_{i j}$ has been described before in (2.12),

$$
h_{i j}=\frac{A_{r} \cos <\overrightarrow{r_{i j}}, \overrightarrow{n_{j}}>}{2 \pi d_{i j}^{2}}(m+1) \cos ^{m}<\overrightarrow{r_{i j}}, \overrightarrow{l_{i}}>
$$

Using the MISO technique, the received signal is given by

$$
\mathbf{y}=\mathbf{H}^{T} \mathbf{x}+\mathbf{n}
$$

where $\mathbf{y}=\left(y_{1}, y_{2}, \ldots y_{K}\right)^{T}, y_{k}$ represents the signal received by user $k, \mathbf{x}=\left(x_{1}, x_{2}, \ldots x_{Q}\right)^{T}$, $x_{k}$ is the transmitted signal from transmitter $k, \mathbf{n}=\left(n_{1}, n_{2}, \ldots n_{K}\right)^{T}$, and $n_{k}$ is the additive noise at receiver $k$. The noise here we model is the thermal noise and background noise (shot noise is assumed small). 


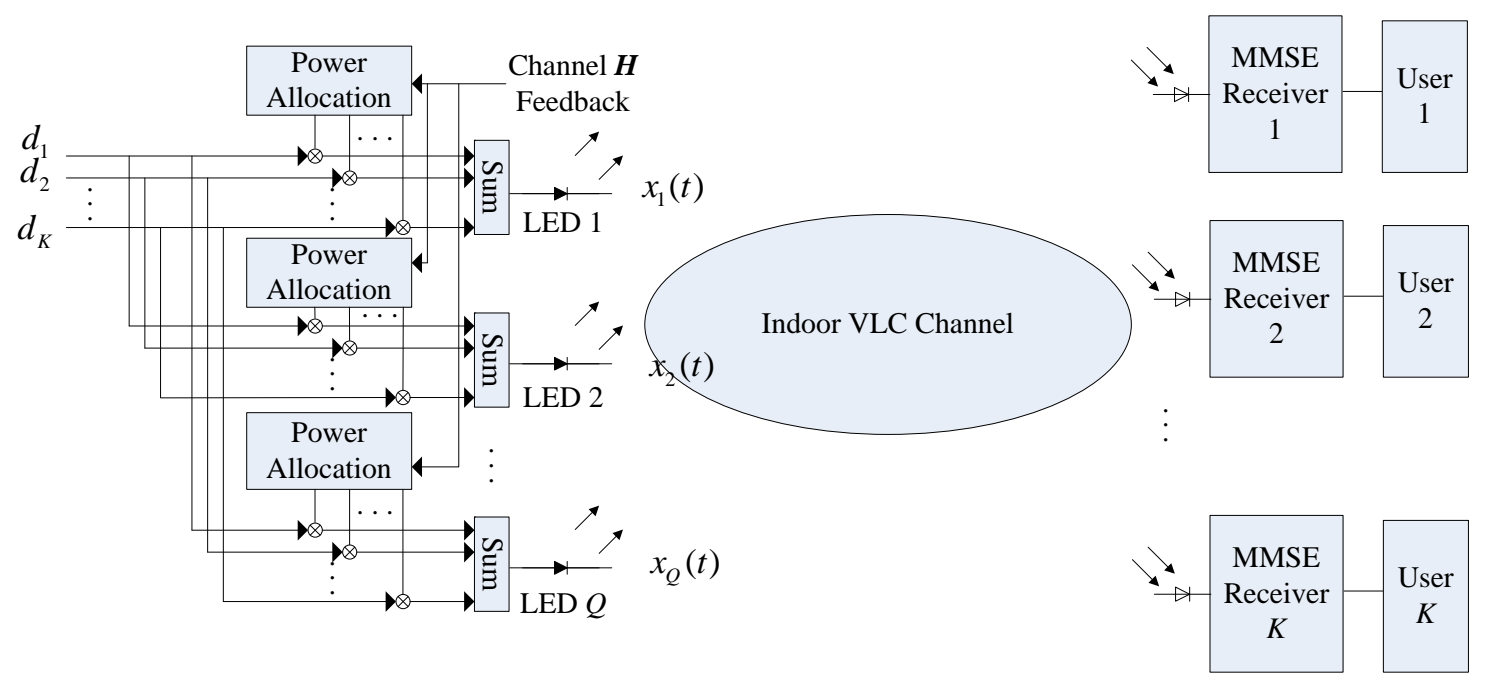

Figure 4.3: Block diagram of the proposed PAJO algorithm to support $K$ users simultaneously.

\subsection{Power Allocation Joint Optimization}

A power allocation joint optimization algorithm is proposed in this section. According to this algorithm, we maximize the minimum SINR of all the users and design a MMSE receiver for each user. A block diagram of the PAJO algorithm is shown in Figure 4.3. For PAJO, the transmitted signal from each LED depends on $d_{1}$ to $d_{K}$ as shown in this figure. $d_{k}$ is the intended data for user $k$. The power allocation for each LED is different, which depends on the channel feedback from the users. Since the location of each user is different, the channels of the different users are different. Therefore, we need to allocate the transmitted power to compensate the channel loss for different users.

\subsubsection{Multiuser MISO System Description}

In this subsection, we assume that the VLC network has $Q$ LEDs with $K$ users, and the VLC channel between LED $q$ and user $k$ is characterized by $h_{q k}$ in (4.4). Let 
$s_{k}(t)$ be the signal that is intended for user $k$ at the given symbol time. The $q$ th LED sends a combination of the users' data as

$$
x_{q}(t)=\sum_{k=1}^{K} P_{q k} s_{k}(t)
$$

where $P_{q k} \in\left[0, P_{0}\right]$ is the power of the $q$ th LED allocated to transmitting the data of user $k$. Assuming a total radiation power limit of $P_{0}$ for each LED, which is the maximum optical power radiated from each LED, the constraint $\sum_{k=1}^{K} P_{q k} \leq P_{0}$ needs to be applied on the allocated powers. Since the power of light is nonnegative, we can set up the constraint as

$$
\begin{gathered}
\sum_{k=1}^{K} P_{q k} \leq P_{0} \\
P_{q k} \geq 0, \forall q
\end{gathered}
$$

The signal of the intended data to user $k$ for power allocation can be defined as

$$
s_{k}(t)=\sum_{l=1}^{L} d_{k} c_{k}[l] \cdot G\left(t-l T_{c}\right)
$$

where $\mathbf{c}_{k}$ is the CDMA code for user $k, \mathbf{c}_{k}=\left(c_{k}[1], c_{k}[2] \ldots c_{k}[L]\right)^{T}$. The length of the CDMA code is $L . d_{k}$ is the transmitted data for user $k$, and $G(t)$ is considered to be a rectangular function in this work.

$$
G(t)= \begin{cases}1 & 0<t \leq T_{c} \\ 0 & \text { otherwise }\end{cases}
$$

The signal received by user $k$ can be written as

$$
r_{k}(t)=\sum_{q=1}^{M} h_{q k} x_{q}(t)+n_{k}(t), \quad k=1, \ldots, K
$$


where $n_{k}(t)$ is the additive white noise to the received signal of user $k$. And we assume that the noise is a sum of thermal noise, background noise, and shot noise (which is negligible as described in Chapter 3). After chip matched filtering and sampling the received signal, the discrete time signal received by user $k$ can be written as

$$
r_{k}[l]=\sum_{q=1}^{Q} h_{q k} x_{q}[l]+n_{k}[l], \quad k=1, \ldots, K
$$

After the receiver and the MMSE filter $\mathbf{w}_{k}$, the signal for user $k$ can be represented as

$$
y_{k}=\sum_{l=1}^{L} r_{k}[l] w_{k}[l]+\sum_{l=1}^{L} w_{k}[l] n_{k}[l]
$$

where the MMSE filter for user $k$ can be defined as $\mathbf{w}_{\mathbf{k}}=\left(w_{k}[1], w_{k}[2], \cdots w_{k}[L]\right)^{T}$.

Substituting (4.6), (4.8) and (4.11) into (4.12), we can obtain

$$
y_{k}=\sum_{l=l}^{L} \sum_{j=1}^{K} \sum_{i=1}^{Q} h_{i k} P_{i j} d_{j} c_{j}[l] w_{k}[l]+\sum_{l=1}^{L} w_{k}[l] n_{k}[l]
$$

which can be written in a matrix form as

$$
y_{k}=\mathbf{d}^{T} \mathbf{B}_{\mathbf{k}} \mathbf{C w}_{\mathbf{k}}+\mathbf{n}_{\mathbf{k}}^{\mathbf{T}} \mathbf{w}_{\mathbf{k}}
$$

where $\mathbf{d}$ is the data vector, $\mathbf{d}=\left(d_{1}, d_{2}, \ldots, d_{K}\right)^{T}, \mathbf{n}_{\mathbf{k}}=\left(n_{k}[1], n_{k}[2], \cdots, n_{k}[L]\right)^{T}$. For the optical signal, in this chapter we assume the intended data for each user is a binary data stream $d_{k} \in\{0,1\}$. C represents the CDMA code matrix, which can be represented as 


$$
\mathbf{C}=\left(\begin{array}{cccc}
c_{1}[1] & c_{1}[2] & \cdots & c_{1}[L] \\
c_{2}[1] & c_{2}[2] & \cdots & c_{2}[L] \\
\vdots & \vdots & \ddots & \vdots \\
c_{K}[1] & c_{K}[2] & \cdots & c_{K}[L]
\end{array}\right)
$$

The matrix $\mathbf{B}_{k}=\operatorname{diag}\left(\mathbf{h}_{k}^{T} \cdot \mathbf{P}\right)$, where $\mathbf{h}_{k}=\left(h_{1 k}, h_{1 k}, \cdots, h_{Q k}\right)^{T}$. Thus,

$$
\mathbf{B}_{\mathbf{k}}=\left(\begin{array}{cccc}
\sum_{j} h_{k j} P_{1 j} & 0 & \cdots & 0 \\
0 & \sum_{j} h_{k j} P_{2 j} & \cdots & \vdots \\
\vdots & \cdots & \ddots & 0 \\
0 & 0 & \cdots & \sum_{j} h_{k j} P_{K j}
\end{array}\right)
$$

When considering the MMSE receiver design and maximizing the minimum SINR for each user, the mean-squared error $J_{k}$ for user $k$ is defined as

$$
\begin{aligned}
J_{k} & =E_{d, n}\left\{\left(y_{k}-d_{k}\right)^{2}\right\} \\
& =E_{d, n}\left\{\left(\mathbf{d}^{T} \mathbf{B}_{\mathbf{k}} \mathbf{C} \mathbf{w}_{\mathbf{k}}+\mathbf{n}_{\mathbf{k}}^{\mathbf{T}} \mathbf{w}_{\mathbf{k}}-d_{k}\right)^{2}\right\},
\end{aligned}
$$

where $E_{d, n}$ represents expectation with respect to the data vector $\mathbf{d}$ and the noise $\mathbf{n}_{\mathbf{k}}$.

Let

$$
\frac{\partial J_{k}}{\partial \mathbf{w}_{\mathbf{k}}}=0
$$


which means,

$$
\begin{gathered}
\frac{\partial J_{k}}{\partial w_{k}[1]}=0 \\
\frac{\partial J_{k}}{\partial w_{k}[2]}=0 \\
\vdots \\
\frac{\partial J_{k}}{\partial w_{k}[L]}=0
\end{gathered}
$$

Through some algebraic manipulation, the MMSE linear receiver $\mathbf{w}_{\mathbf{k}}$ can be obtained as

$$
\mathbf{w}_{\mathbf{k}}=\left(\mathbf{C}^{T} \mathbf{B}_{\mathbf{k}} \boldsymbol{\Sigma}_{\mathbf{d}} \mathbf{B}_{\mathbf{k}} \mathbf{C}+\sigma^{2} \mathbf{I}\right)^{-1} \mathbf{C}^{T} \mathbf{B}_{\mathbf{k}} \mathbf{q}_{\mathbf{k}}
$$

where $\boldsymbol{\Sigma}_{\mathbf{d}}$ is the variance matrix for the data, $\boldsymbol{\Sigma}_{\mathbf{d}}=E\left\{\mathbf{d d}^{T}\right\}, \mathbf{q}_{\mathbf{k}}=E\left\{\mathbf{d}^{T} \cdot d_{k}\right\}$, I is the identity matrix with size $L \times L$, and $\sigma^{2}$ is the noise variance from (3.15). Here we consider the noise is white Gaussian noise, equal for all symbols (dominated by thermal noise).

From (4.13), the signal after the receiver can be rewritten as three terms. They are the target (the intended data) for user $k$, the multiple access interference, and the noise. Thus, the signal after the receiver can be represented as

$$
y_{k}=\underbrace{\sum_{l=1}^{L} \sum_{i=1}^{Q} h_{i k} P_{i j} d_{k} c_{k}[l] w_{k}[l]}_{\text {Target }}+\underbrace{\sum_{l=1}^{L} \sum_{\substack{j=1 \\ j \neq k}}^{K} \sum_{i=1}^{Q} h_{i k} P_{i j} d_{j} c_{j}[l] w_{k}[l]}_{\text {Interference }}+\underbrace{\sum_{l=1}^{L} w_{k}[l] n_{k}[l]}_{\text {Noise }},
$$

which can be written in matrix form as

$$
y_{k}=\underbrace{\mathbf{d} \widehat{\mathbf{E}}_{\mathbf{k}} \mathbf{B}_{\mathbf{k}}^{T} \mathbf{C w}_{\mathbf{k}}}_{\text {Target }}+\underbrace{\mathbf{d}\left(\mathbf{I}-\widehat{\mathbf{E}}_{\mathbf{k}}\right) \mathbf{B}_{\mathbf{k}}^{T} \mathbf{C w}_{\mathbf{k}}}_{\text {Interference }}+\underbrace{\mathbf{n}_{\mathbf{k}} \mathbf{w}_{\mathbf{k}}}_{\text {Noise }},
$$


where

$$
\widehat{\mathbf{E}}_{\mathbf{k}}=\left(\begin{array}{cccc}
0 t h \downarrow & \\
0 & 0 & \cdots & 0 \\
0 & 0 & \cdots & 0 \\
\vdots & \vdots & 1 & \vdots \\
0 & 0 & \cdots & 0
\end{array}\right) \leftarrow k t h
$$

Therefore, the SINR for user $k$ can be written as

$$
\operatorname{SINR}_{\mathrm{k}}=\frac{\mathbf{w}_{\mathbf{k}}^{T} \mathbf{C}^{T} \mathbf{B}_{\mathbf{k}} \widehat{\mathbf{E}}_{\mathbf{k}} \boldsymbol{\Sigma}_{\mathbf{d}} \widehat{\mathbf{E}}_{\mathbf{k}} \mathbf{B}_{\mathbf{k}} \mathbf{C w}_{\mathbf{k}}}{\mathbf{w}_{\mathbf{k}}^{T} \mathbf{C}^{T} \mathbf{B}_{\mathbf{k}} \widehat{\mathbf{A}}_{\mathbf{k}} \boldsymbol{\Sigma}_{\mathbf{d}} \widehat{\mathbf{A}}_{\mathbf{k}} \mathbf{B}_{\mathbf{k}} \mathbf{C} \mathbf{w}_{\mathbf{k}}+\sigma^{2} \mathbf{w}_{\mathbf{k}}^{T} \mathbf{w}_{\mathbf{k}}}
$$

where we define $\widehat{\mathbf{A}}_{\mathbf{k}}$ as

$$
\widehat{\mathbf{A}}_{\mathrm{k}}=\mathbf{I}-\widehat{\mathbf{E}}_{\mathbf{k}} .
$$

When maximizing the minimum SINR for each user, the cost function can be defined as

$$
\max _{P} \min _{k} \mathrm{SINR}_{\mathrm{k}}
$$

To simplify the cost function, the $\beta$-fair cost function can be used [31]. We can consider minimizing the sum of the function instead of doing a minmax search when $\beta \rightarrow \infty$ [31]. Thus, (4.26) can be written as

$$
\min _{P} \sum_{k=1}^{K} \frac{\beta+1}{\operatorname{SINR}_{\mathrm{k}}^{\beta+1}} . \quad \beta \rightarrow \infty
$$

In this thesis, we solve this optimization problem assuming $\beta=100$ using the Matlab optimization tool box. 


\subsubsection{Other Power Allocation Systems}

To evaluate the BER performance of PAJO, we compare it to other power allocation and receiver algorithms. In this subsection, three types of power allocation and receiver systems are described.

The first one is a fair power allocation system with the MMSE receiver. It is denoted as "FP". In this scheme, the power allocated to each user signal is the same. To minimize the interference, the MMSE receiver is employed.

The second system assumes optimal power allocation with the correlation receiver (the filter of user $k$ matched to $\mathbf{c}_{k}$ ) instead of the MMSE receiver. It is denoted as "MF". In this system, to maximize the minimum SINR of each user, we optimize the power allocation only.

The last type of system is the fair power allocation system with correlation receiver. It is denoted as "FF". In this system, the power allocated to each user signal is the same and the correlation receiver is used at the users.

\subsubsection{Illumination Requirements}

For applications that need both data transmission and illumination requirements, we can use our PAJO algorithm to find the optimal power allocation scheme and the MMSE receivers. Usually, users in the room will need data transmission as well as illumination. Considering the total radiation power limit and the illumination 
requirements of the users; the constraints can be represented as

$$
\left\{\begin{array}{l}
\sum_{k=1}^{K} P_{q k} \leq P_{o} \\
P_{q k} \geq 0 \\
\left|\sum_{q=1}^{Q} \sum_{j=1}^{K} h_{q k} P_{q j} \eta-P_{r k}\right| \leq \Delta \\
k=1,2, \ldots K
\end{array}\right.
$$

where $P_{r k}$ is the required received power for illumination of user $k$, and $\Delta$ is the illumination tolerance. This constraint is to make sure that the illumination level at these users will not be too dark or too bright. $\eta$ represents the CDMA code weight to CDMA code length ratio, which decides the illumination level.

To illuminate the room uniformly in space, we define virtual users that must have a fixed illumination, but no data transmission. These $E$ users are distributed uniformly in the room. For these virtual users that require just illumination, we use the constraint

$$
\left|\sum_{q=1}^{Q} \sum_{j=1}^{E} h_{q e} P_{q j} \eta-P_{r e}\right| \leq \Delta \quad e=1,2, \ldots E
$$

where $h_{q e}$ is the channel between the $q$ th transmitter to the eth virtual user, $P_{r e}$ is the required received power illumination for user $e$, and $E$ is the number of virtual users that only need illumination.

\subsubsection{Numerical Results of PAJO}

To get the numerical results, the parameters assumed are shown in Table 4.2. 
Table 4.2: Parameters of Numerical Results of PAJO

\begin{tabular}{cc}
\hline size of the room & $5 \mathrm{~m} \times 5 \mathrm{~m} \times 3 \mathrm{~m}$ \\
positions of the LED lamps & $(1.25,1.25,3)(1.25,3.75,3)$ \\
& $(3.75,1.25,3)(3.25,3.75,3)$ \\
positions of the users & $(1.5,2.0,0)(2.5,2.5,0)$ \\
radiation power of each lamp & $(1.2,1.2,0)(4.5,2.0,0)$ \\
OOC code index & $300 \mathrm{~mW}$ \\
& $\{1,2,4\}\{2,3,5\}$ \\
length of the code & $\{3,4,6\}\{4,5,7\}$ \\
& $72 \%$ \\
semiangles of the 1-LED case & $20^{\circ}$ \\
semiangles of the 25-LED case & $20^{\circ}$ \\
\hline
\end{tabular}

The simulated BER for one to four users for the 1-LED lamp case without illumination requirements is plotted in Figure 4.4. The calculated BER is the average bit error rate of all users. In this figure, the proposed PAJO algorithm is compared with other power allocation system as described in Section 4.3.2., where "JO" denotes PAJO, "FP" represents the fair power allocation with MMSE receiver case, "MF" is the case of optimal power allocation with the correlation receiver (matched filter) and "FF" represents fair power allocation with the correlation receiver case. According to these results, our proposed algorithm always has the best BER performance among all techniques for one to four users. Note that in both Figures 4.4 and 4.5, assuming a MMSE receiver is less important that optimizing the power among users.

Similar results can be observed in the 25-LED case. In Figure 4.5, PAJO has the 


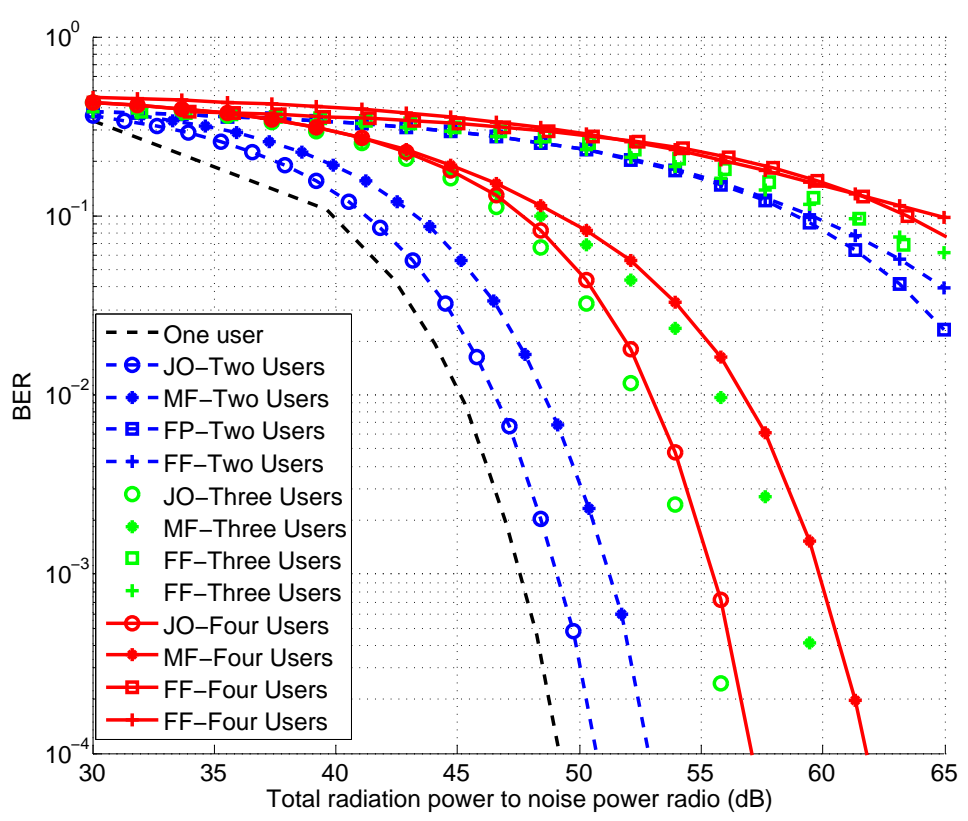

Figure 4.4: BER of 1-LED lamp case with 1 to 4 users for 4 lamps, no illumination requirements, semiangle $=20^{\circ}$

best BER performance for one to four users. Because of more MAI for more users, the BER performance of PAJO for the two users case is better than the three users and four users cases.

The BER performance of the proposed PAJO technique compared with the block diagonalization algorithm precoding MISO system proposed in [13] is shown in Figure 4.6 for the case of no illumination requirement. For this simulation, two, three and four users are supported in the indoor VLC system, "BD" represents the block diagonalization algorithm, and "JO" represents the PAJO algorithm. According to the results in Figure 4.6, the BER of PAJO proposed in this chapter is better than the algorithm in [13]. Since the algorithm proposed in [13] adds a DC bias to the precoded transmitted signals, the distance between the constellation points of the transmitted signal is compressed by the total radiation power limit. But for PAJO, the transmitted power is allocated optimally; there is no need to add a DC bias. Therefore, the PAJO algorithm has a better BER performance. 


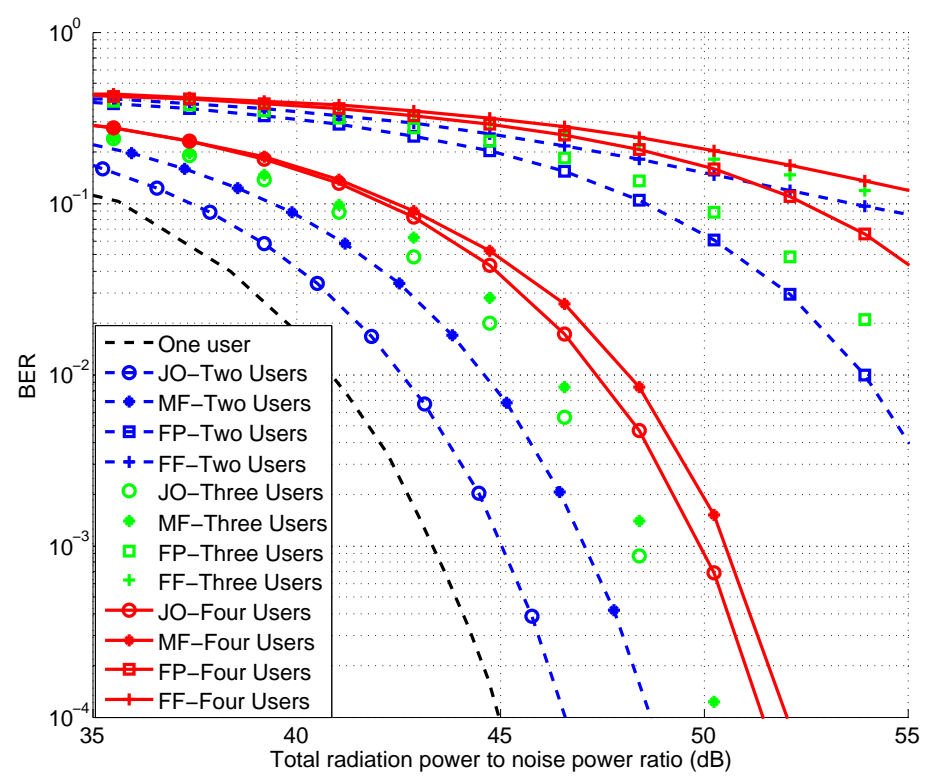

Figure 4.5: BER of 25-LED lamp case with 1 to 4 users for 4 lamps, no illumination requirements, semiangle $=20^{\circ}$

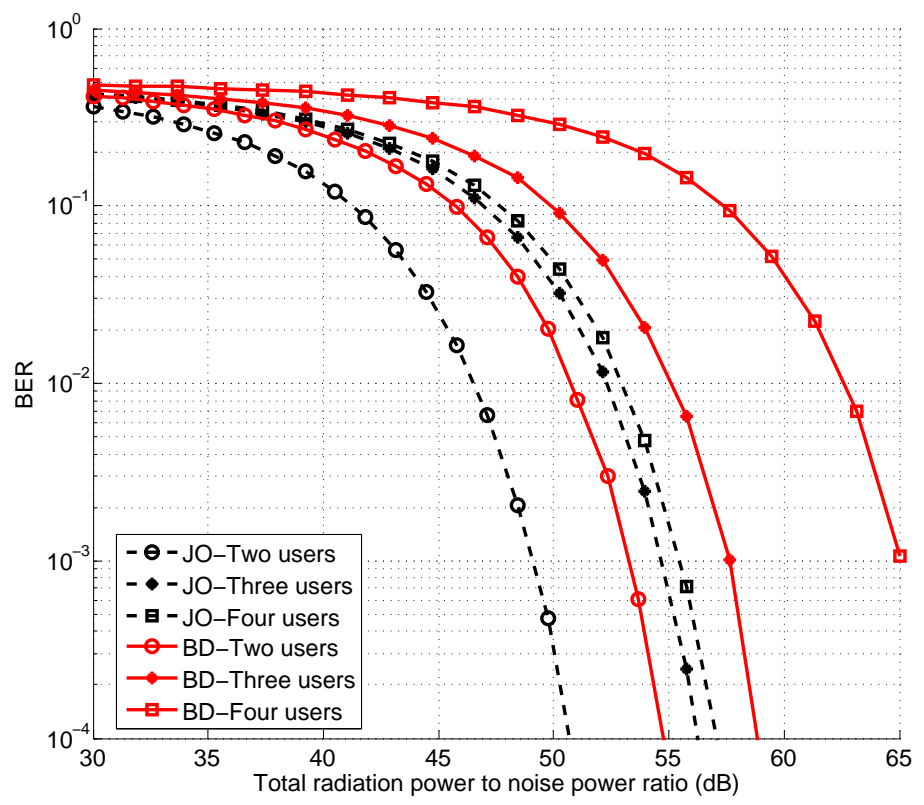

Figure 4.6: BD algorithm vs PAJO for 4, 3 and 2 users with 1-LED lamp case, no illumination requirements, semiangle $=20^{\circ}$

The illumination requirement for a the standard office environment should be 400 lx, determined by the Illuminating Engineering Society of North America [23]. Since 
for a detector with $1 \mathrm{~cm}^{2}$ effective area, $1 \mathrm{~lx}$ is equal to $10^{-4} \mathrm{~mW}$, we set up the required received power by users as $P_{r k}=P_{r e}=0.04 \mathrm{~mW}(400 \mathrm{~lx})$. We consider the illumination constraints to compare the BER performance of 1-LED and 25-LED cases. We assume $E=16$ virtual users needing just illumination, distributed in a rectangular grid around the room. For the 25-LED lamp cases, we assume the total transmitted power for each lamp is the same as 1-LED lamp case. The BER for the 1-LED lamp and 25-LED lamp cases with and without illumination constraints can be seen in Figure 4.7 for the PAJO method. According to the results in Figure 4.7, the BER with illumination requirements for the 1-LED and 25-LED lamp cases are always worse than without illumination requirements. Furthermore, the 25-LED lamp case has better performance than the 1-LED lamp case.

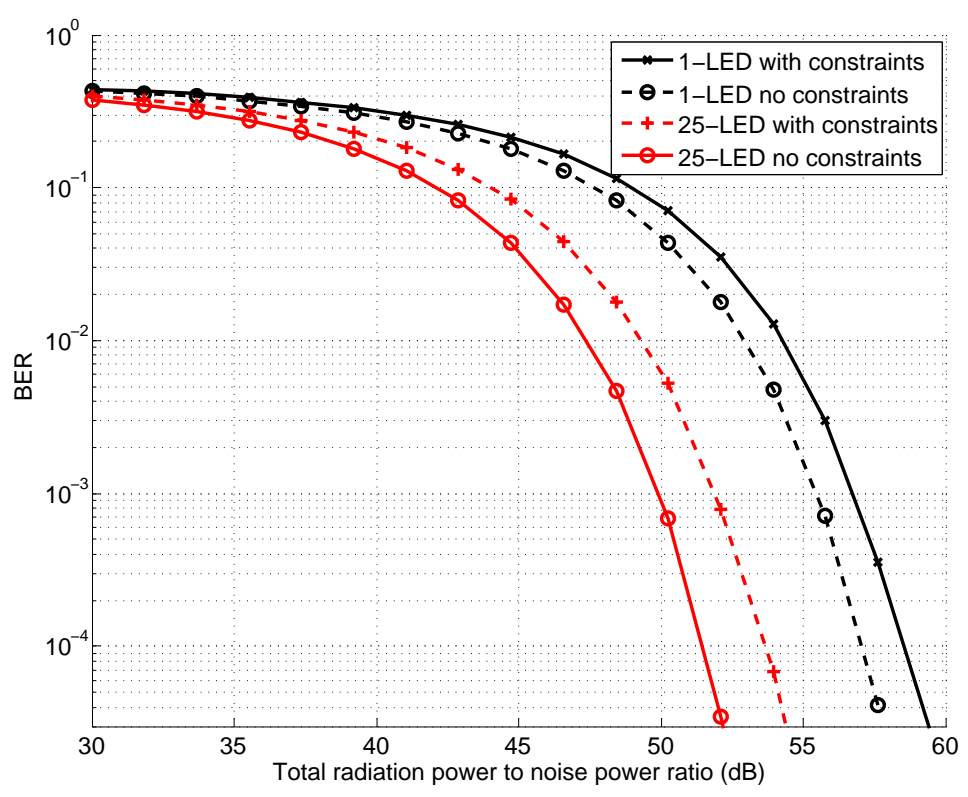

Figure 4.7: BER of 4 users for 1-LED lamp case and 25-LED lamp cases with and without illumination requirements, the illumination is $400 \mathrm{~lx}$ with $10 \%$ tolerance

Figure 4.8-(a) shows the contour plot of the illumination distribution for 4 users with both data transmission and illumination requirements, plus 16 virtual users with illumination requirements only. Figure 4.8-(b) shows the illumination distri- 
bution without data transmission requirements. Comparing these two figures, the illumination distribution in (a) is still smooth and flat. That is to say, the illumination constraints makes the illumination in the room not too dark and not too bright, and the illuminations at all the users' locations is satisfied.

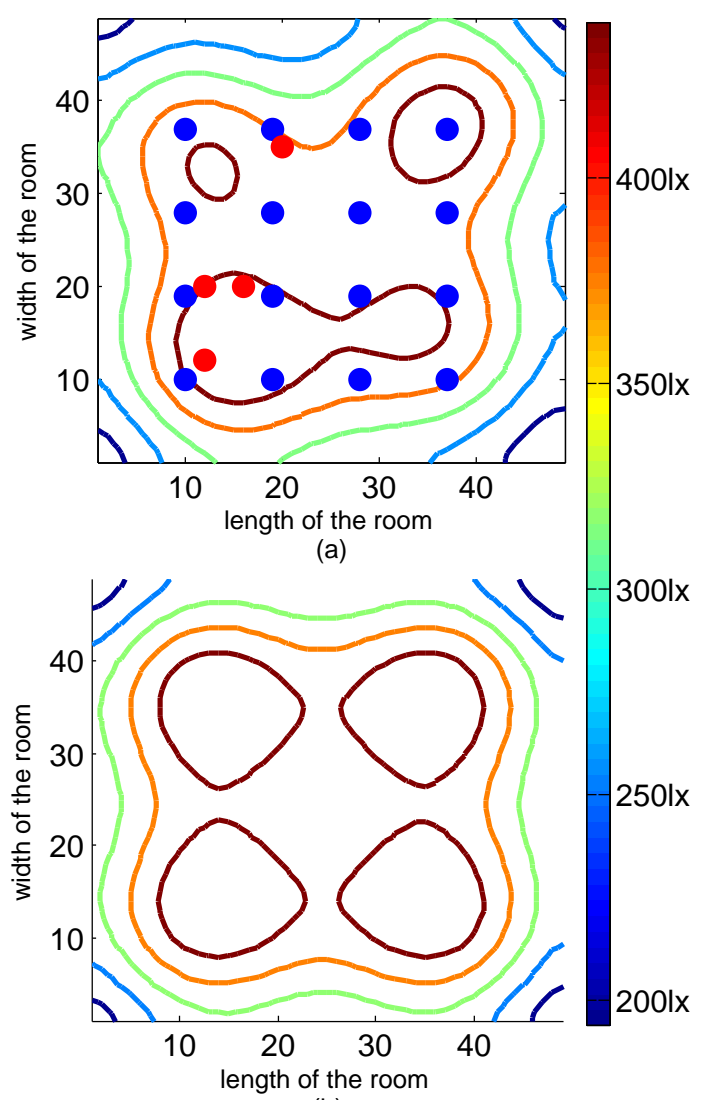

(b)

Figure 4.8: Illumination distribution comparison of (a) data transmission case and (b) no data transmission case. The red dots identify the real users, and the blue dots represent the virtual users with $10 \%$ tolerance, semiangle $=20^{\circ}$, and the 25 -LED model.

The illumination tolerance $\Delta$ decides the illumination performance in indoor VLC systems. In addition, it also affects the BER performance for multiuser communications. The tolerance to $P_{r e}$ we used varies from $9 \%$ to $60 \%$. From the simulation results, we observe that if the tolerance of illumination increases, the BER performance curve converges to the no illumination constraint case. Simulations are shown 
for 4 users with data and illumination requirements and 16 users with only illumination requirement in Figure 4.9. From this result, the BER with $60 \%$ tolerance is quite close to the BER without constraints. Note that this variation in the room lighting may not be acceptable from a human perception perspective. The evaluation of this aspect of the design is beyond the scope of this thesis.

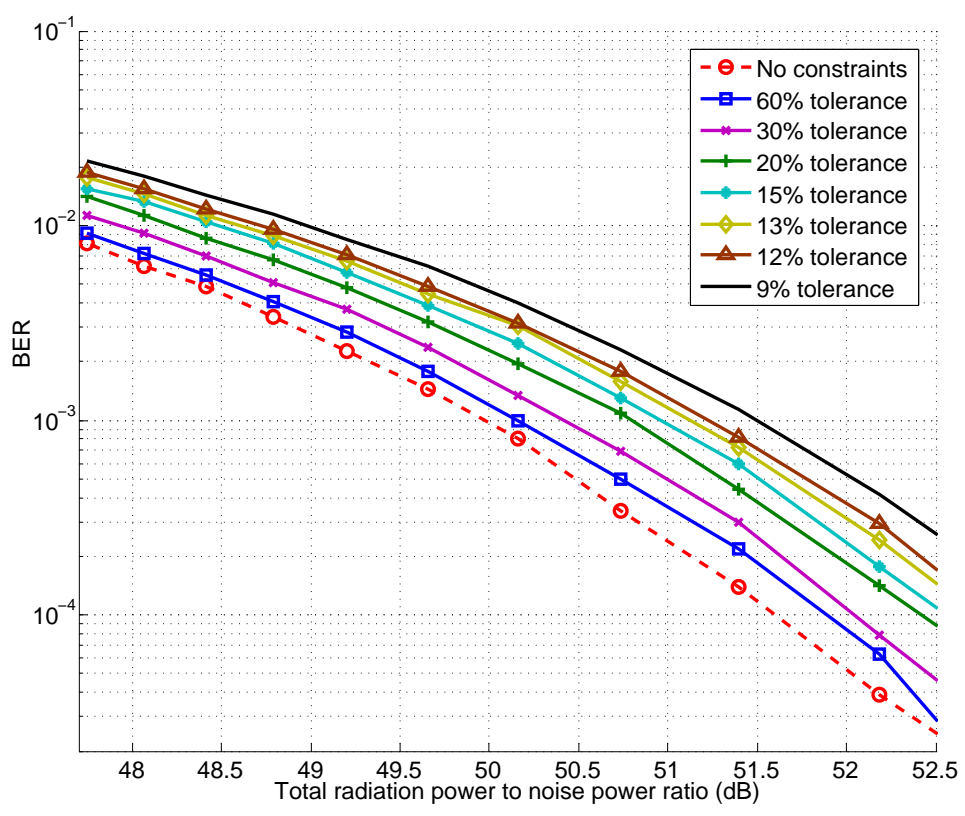

Figure 4.9: BER comparison of different tolerance with 4 users and 16 virtual users for the 25-LED lamp case, $400 \mathrm{~lx}$ illumination requirements

The LED semiangle is another significant parameter we need to consider in indoor VLC systems, since the semiangle decides the beamwidth and power distributions on the floor. It can also affect the BER performance. Figure 4.10 shows the BER with $5^{\circ}, 12^{\circ}$ and $20^{\circ}$ semiangles. According to these results, the BER curves can be divided into three groups with respect to the three semiangles. We can clearly observe that the $20^{\circ}$ semiangle has the worst performance among the three groups. For the cases we tried, the semiangle is the dominant parameter affecting the BER performance, compared with the illumination tolerance. But for other users' locations and different geometries, we may see different results. 


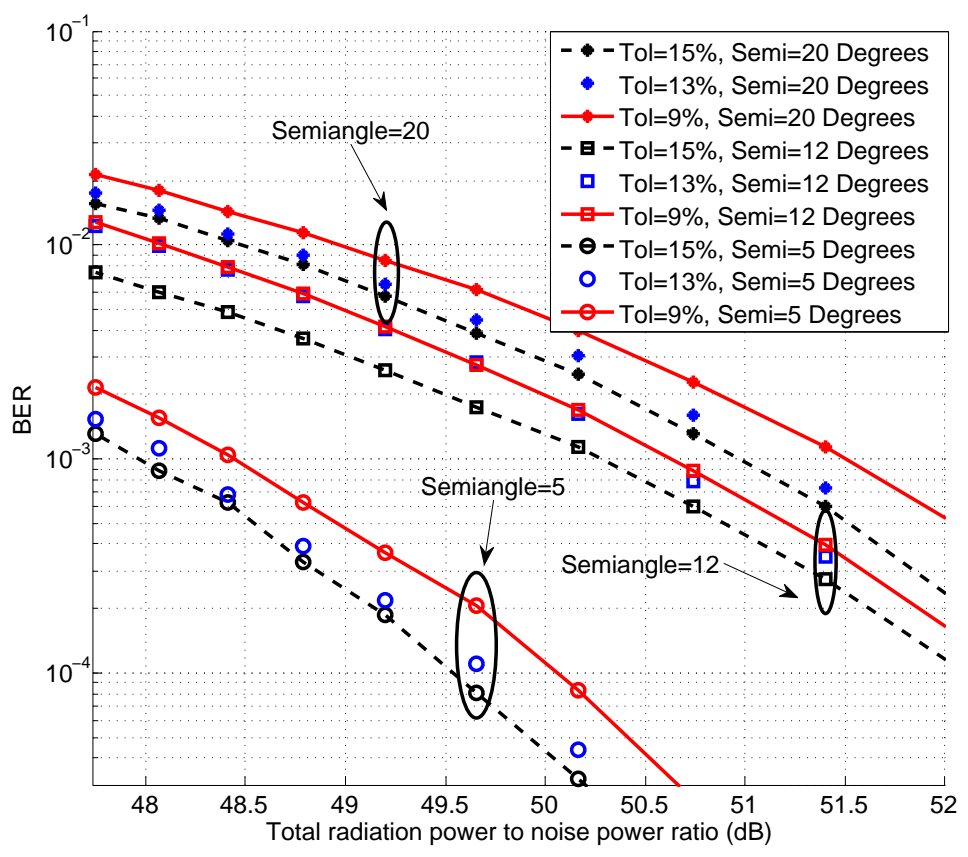

Figure 4.10: BER comparison of different semiangles and different tolerance with 4 users and 16 virtual users for 25-LED lamp case, 400 lx illumination requirements

Introducing virtual users to regulate the illumination level can have a detrimental effect on communications performance, expecially if the illumination tolerance is too low. Figure 4.11 shows the BER performance with 4 users needing communication and illumination and some virtual users who need illumination only. In this case, we compare 4, 10 and 16 virtual users who need illumination only. From the results, the more virtual users in an indoor VLC system, the worse the BER performance. We suspect that this effect comes from the fact that as $E$ increases some virtual users get close to the edges of the room, which are costly to illuminate. 


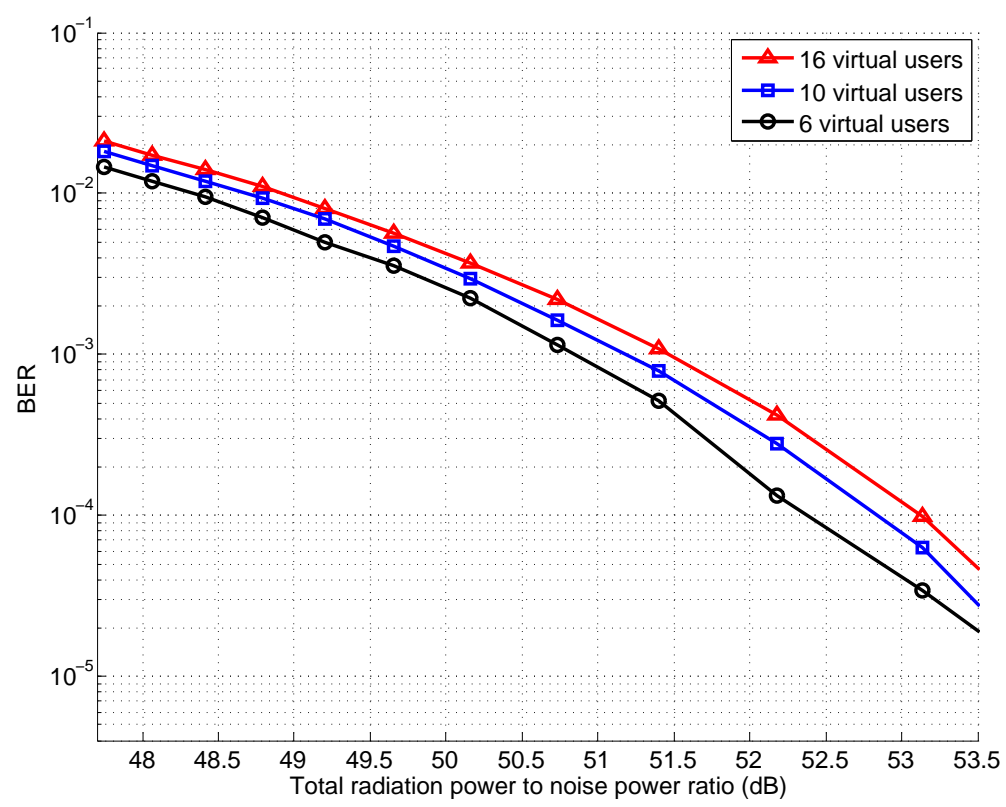

Figure 4.11: BER comparison of different number of virtual users for 25-LED lamp case, $400 \mathrm{~lx}$ illumination requirements and $10 \%$ tolerance

The choice of CDMA code can also result in a different BER performance. In Figures 4.4-4.11 we used a simple OOC code. In Figures 4.12, we employ the coded multilevel expurgated pulse position modulation (C-MEPPM) scheme to support the 4 users in the indoor VLC system [24]. The MEEPM modulation is a multilevel scheme that improves upon the M-PAM scheme discussed in Chapter 3. The interested readers can refer to [24] for details on the C-MEPPM technique. One of the advantage of the C-MEPPM code is that it can provide better average illumination on the floor than the OOC code, since for OOC, the percentage of pulse to blank ratio is relatively low. For example, for the OOC code with code index $\{1,2,4\}$ and length 7 , the pulse to blank ratio $\eta$ is only $42 \%$. That is to say, the optical power that users can use is only $42 \%$ of the maximum optical power. However, the C-MEPPM code can improve this ratio to almost $50 \%$. At the same time, employing C-MEPPM can improve the BER performance. In Figure 4.12, two kinds of C-MEPPM are used, length 13 and 63. According to the results, C-MEPPM has better BER performance 
than the OOC code in VLC systems, and the longer C-MEPPM code length can provide better BER performance. For this result we did not impose any illumination constraints.

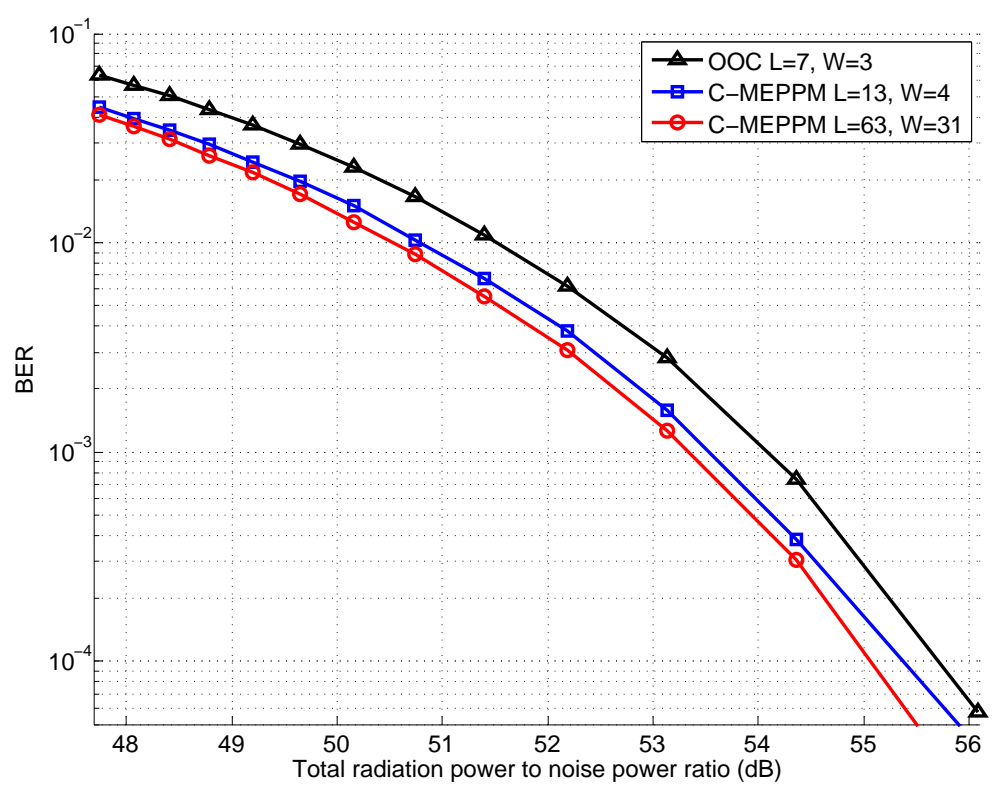

Figure 4.12: BER of OOC vs C-MEPPM without illumination constraints

\subsection{Chapter Summary}

To design a real system for the multiuser case without illumination constraints using the PAJO algorithm, we can set parameters and get the performance as shown in Table 4.3. From this table, the BER of the 25-LED case for these users locations is lower than that of the 1-LED case.

For the case with illumination constraints, we assume that there are 16 virtual users distributed uniformly around the room. The required illumination power for all the users is $0.04 \mathrm{~mW}(400 \mathrm{~lx})$. The illumination tolerance is $9 \%$. From that point of view, the BER for the 25-LED case can reach $10^{-4}$ when the data rate is $28.57 \mathrm{Mb} / \mathrm{s}$ (with length 7 OCDMA code). 
Table 4.3: Parameters of real design

\begin{tabular}{|c|c|}
\hline size of the room & $5 \mathrm{~m} \times 5 \mathrm{~m} \times 3 \mathrm{~m}$ \\
\hline positions of the LED lamps & $(1.25,1.25,3)(1.25,3.75,3)$ \\
\hline & $(3.75,1.25,3)(3.25,3.75,3)$ \\
\hline \multirow[t]{2}{*}{ positions of the users } & $(1.5,2.0,0)(2.5,2.5,0)$ \\
\hline & $(1.2,1.2,0)(4.5,2.0,0)$ \\
\hline radiation power of each lamp & $300 \mathrm{~mW}$ \\
\hline \multirow[t]{2}{*}{ OOC code index } & $\{1,2,4\}\{2,3,5\}$ \\
\hline & $\{3,4,6\}\{4,5,7\}$ \\
\hline length of the code & 7 \\
\hline$\eta$ & $42 \%$ \\
\hline semiangles of the 1-LED case & $20^{\circ}$ \\
\hline semiangles of the 25-LED case & $20^{\circ}$ \\
\hline modulation scheme & 2-PAM \\
\hline data rate & $28.57 \mathrm{Mbit} / \mathrm{s}$ \\
\hline BER for 1-LED case & $10^{-3}$ \\
\hline BER for 25-LED case & $10^{-7}$ \\
\hline
\end{tabular}




\section{Chapter 5}

\section{Conclusions and Future Work}

\subsection{Conclusions}

In recent years, high-speed indoor optical networks using LEDs have been studied with advantages in low power consumption, high on-off frequency, and eye safety. For future indoor networks such as offices and homes, visible light communications are attractive. The research we performed is summarized as follows.

- We analyzed the characteristics of the indoor VLC model, and propose two kinds of transmitter models. They are the 1-LED and 25-LED lamp models. For these two models, we compare the illumination performance and model the impulse response.

- For a single user indoor VLC system, a M-PAM modulation scheme is employed. We compare the BER of 2-PAM, 4-PAM and 8-PAM. According to the simulation results, the 2-PAM has the best BER performance but 8-PAM has the best bandwidth efficiency.

- A PAJO algorithm is proposed in this thesis for the multiuser case. It maximizes 
the minimum SINR of all the users and employs a MMSE receiver for each user. Compared to the algorithm in [13], our proposed algorithm has better BER performance. Our proposed PAJO can also satisfy the illumination requirements in an indoor VLC system.

\section{$5.2 \quad$ Future Work}

Many extensions to this work can be made. We need to consider the effects of the LED nonlinear response and transmit driver complexity on the PAJO algorithm. To support high speed data transmission in VLC system, we also need to consider the inter symbol interference (ISI). To eliminate the ISI, channel equalization, orthogonal frequency division multiplexing (OFDM), or the application of a MIMO-OFDM technique in indoor VLC should be considered. 


\section{Bibliography}

[1] Z. Ghassemlooy, W. Popoola, Rajbhandari, Optical Wireless Communications:System and Channel Modeling with Matlab, CRC Press, 2013.

[2] Y. Tanaka, S. Haruyama, M. Nakagawa, "Wireless Optical Transmissions with White Wireless Home Links," The 11th IEEE International Symposium on Indoor and Mobile Radio Communications, vol. 2, pp: 1325-1329, Sep. 2000.

[3] Y. Tanaka, K. Toshihiko, S. Haruyama, "Indoor Visible Communication Utilizing Plural White LEDs as Lighting," 2001 12th IEEE International Symposium on Personal Indoor and Mobile Radio Communications, vol. 2, pp: F-81-F-85, 2001.

[4] K. Toshihiko, M. Nakagawa, "Integrated System of White LED Visible-Light Communication and Power-Line Communication," IEEE Transactions on Consumer Electronics, vol. 49, no. 1, pp: 71-79, 2003.

[5] K. Toshihiko, M. Nakagawa, "Fundamental Analysis for Visible Light Communication System Using LED Lights," IEEE Transactions on Consumer Electronics, vol. 50, no. 1, pp: 100-107, 2004.

[6] K. Toshihiko , S. Haruyama, M. Nakagawa, "Performance evaluation of narrowband OFDM on integrated system of power line communication and visible light wireless communication," 2006 1st International Symposium on Wireless Pervasive Computing, 2006. 
[7] G. Agrawal, Rajbhandari, Fiber-Optic Communication Systems, A JOHN WILEY and SONS, INC., PUBLICATION, 2013.

[8] D. Buso, S. Bhosle, Y. Liu, M. Ternisien, "OLED Electrical Equivalent Device for Driver Topology Design," IEEE Transactions on Industry Applications, vol. 50, no. 2, pp: 1459-1468, 2014.

[9] M. Martin-Arias, N. Huerta-Medina, M. Rico-Secades, "Using wireless technologies in Lighting Smart Grids," 2013 International Conference on New Concepts in Smart Cities: Fostering Public and Private Alliances (SmartMILE), pp: 1-6, 2013.

[10] D. Takase, T. Ohtsuki, "Optical wireless MIMO communications," Global Telecommunications Conference 2004, vol. 2, pp: 928-932, 2004.

[11] R. Mesleh, R. Mehmood, H. Elgala, H. Haas, "Indoor MIMO Optical Wireless Communication Using Spatial Modulation," 2010 IEEE International Conference on Communications (ICC), pp: 1-5, 2010.

[12] S. Dissanayake, J. Armstrong, "Comparison of ACO-OFDM, DCO-OFDM and ADO-OFDM in IM/DD Systems," Journal of Lightwave Technology, vol. 31, no. 7, pp: 1063-1072, 2013.

[13] Y. Hong, J. Chen, Z. Wang, C. Yu, "Performance of a Precoding MIMO System for Decentralized Multiuser Indoor Visible Light Communications," IEEE Photonics Journal, vol. 5, no. 4, 2013.

[14] Qianling Cao, M. Brandt-Pearce, S. Wilson, "Free Space Optical MIMO System Using an Optical Pre-Amplifier," Global Telecommunications Conference 2006, pp: 1-5, 2006. 
[15] J. Kahn, J. Barry, "Wireless infrared communications," Proceedings of the IEEE, vol. 85, no. 2, pp: 265-298, 1997.

[16] C. Xi, A. Mirvakili, V. Koomson, "A Visible Light Communication System Demonstration Based on 16-Level Pulse Amplitude Modulation of an LED Array," 2012 Symposium on Photonics and Optoelectronics (SOPO), pp: 1-4, 2012.

[17] M. Brandt-Pearce, A. Dharap, "Transmitter-Based Multiuser Interference Rejection for the Down-Link of a Wireless CDMA System in a Multipath Environment," IEEE Journal on Selected Areas in Communications, vol. 18, no. 3, pp: 407-417, 2000.

[18] Kyongkuk Cho, Dongweon Yoon, "On the general BER expression of one- and two-dimensional amplitude modulations," IEEE Transactions on Communications, vol. 50, no. 7, pp: 1074-1080, 2002.

[19] B. Vojcic, Won Mee Jang, "Transmitter precoding in synchronous multiuser communications ,"IEEE Transactions on Communications, vol. 46, no. 10, pp: 1346-1355, 1998.

[20] Won Mee Jang, B. Vojcic, R. Pickholtz, "Joint transmitter-receiver optimization in synchronous multiuser communications over multipath channels," IEEE Transactions on Communications, vol. 46, no. 2, pp: 269-278, 1998.

[21] Zhenhua Yu, R. Baxley, G. Zhou, "Multi-user MISO broadcasting for indoor visible light communication," 2013 IEEE International Conference on Acoustics, Speech and Signal Processing (ICASSP), pp: 4849-4853, 2013.

[22] M. Noshad, M. Brandt-Pearce, "Expurgated PPM Using Symmetric Balanced Incomplete Block Designs," IEEE Communications Letters, vol. 16, no. 7, pp: 968$971,2012$. 
[23] M. Noshad, M. Brandt-Pearce, "Application of Expurgated PPM to Indoor Visible Light Communications Part I: Single-User Systems," Journal of Lightwave Technology, vol. 32, no. 5, pp: 875-882, 2013.

[24] M. Noshad, M. Brandt-Pearce, "Application of Expurgated PPM to Indoor Visible Light CommunicationsPart II: Access Networks," Journal of Lightwave Technology, vol. 32, no. 5, pp: 883-890, 2014.

[25] Kwonhyung Lee, Hyuncheol Park, "Channel model and modulation schemes for visible light communications," 2011 IEEE 54th International Midwest Symposium on Circuits and Systems (MWSCAS), vol. 85, no. 2, pp: 1-4, 2011.

[26] http://www.iorodeo.com/content/panels-led-arrays

[27] http://www.diodes.com/datasheets/ds23001.pdf

[28] K. Toshihiko, M. Nakagawa, "Fundamental analysis for visible-light communication system using LED lights," IEEE Transactions on Consumer Electronics, vol. 50, no. 1, pp: 100-107, 2004.

[29] Lubin Zeng, D. O’Brien, Hoa Minh, G. Faulkner, "High data rate multiple input single output (MISO) optical wireless communications using white led lighting," IEEE Journal on Selected Areas in Communications, vol. 27, no. 9, pp: 1654-1662, 2009.

[30] Hooshang Ghafouri-Shiraz, M. Massoud Karbassian, Optical CDMA Priciples, and Applications, WILEY Press, 2012.

[31] Xin Wang, Di Wang, Hanqi Zhuang, "Fair energy-efficient resource allocation in wireless sensor networks over fading TDMA channels," IEEE Journal on Selected Areas in Communications, vol. 28, no. 7, pp: 1063-1072, 2010. 